\title{
ALMOST SURE WELL-POSEDNESS OF THE CUBIC NONLINEAR SCHRÖDINGER EQUATION BELOW $L^{2}(\mathbb{T})$
}

\author{
JAMES COLLIANDER, TADAHIRO OH
}

\begin{abstract}
We consider the Cauchy problem for the one-dimensional periodic cubic nonlinear Schrödinger equation (NLS) with initial data below $L^{2}$. In particular, we exhibit nonlinear smoothing when the initial data are randomized. Then, we prove local wellposedness of NLS almost surely for the initial data in the support of the canonical Gaussian measures on $H^{s}(\mathbb{T})$ for each $s>-\frac{1}{3}$, and global well-posedness for each $s>-\frac{1}{12}$.
\end{abstract}

\section{Contents}

1. Introduction

1.1. Low Regularity Well-Posedness Theory

1.2. Invariant Gibbs Measures

1.3. Almost Sure Local Well-Posedness

1.4. Almost Sure Global Well-Posedness

1.5. Remarks

2. Notation

3. Deterministic and Probabilistic Lemmata

3.1. Deterministic Lemmata

3.2. Probabilistic Lemmata

4. Local Theory

4.1. Basic Setup

4.2. Estimate on $\mathcal{N}_{2}$

4.3. Estimate on $\mathcal{N}_{1}$ : High Modulation Cases

4.4. Estimate on $\mathcal{N}_{1}$ : Low Modulation Cases

5. Global Theory

5.1. Reduction of Theorem 2 to Proposition 5.2

5.2. Basic Setup

5.3. Estimate on $\mathcal{N}_{2}$

5.4. Estimate on $\mathcal{N}_{1}$ : High Modulation Cases

5.5. Estimate on $\mathcal{N}_{1}$ : Low Modulation Cases

References

2010 Mathematics Subject Classification. 35Q55, 37K05, 37L50, 37L40.

Key words and phrases. Schrödinger equation; NLS, well-posedness; invariant measures; ill-posedness. J.C. was supported in part by NSERC grant RGP250233-07. 


\section{INTRODUCTION}

We consider the Cauchy problem for the one-dimensional periodic cubic nonlinear Schrödinger equation (NLS):

$$
\left\{\begin{array}{l}
i u_{t}-u_{x x} \pm u|u|^{2}=0 \\
\left.u\right|_{t=0}=u_{0}, x \in \mathbb{T}=\mathbb{R} / 2 \pi \mathbb{Z}
\end{array}\right.
$$

We first establish almost sure local well-posedness forl (1.1) with respect to the canonical Gaussian measure supported on $H^{s}(\mathbb{T})$ in the range $-\frac{1}{3}<s<0$. Then, we establish almost sure global well-posedness in $H^{s}(\mathbb{T})$ for $-\frac{1}{12}<s<0$. These results are motivated by (a) the well-posedness theory of nonlinear dispersive equations with low regularity initial conditions and (b) construction of measures on phase spaces which are invariant under the (1.1) evolution.

1.1. Low Regularity Well-Posedness Theory. The well-posedness theory for the Cauchy problem (1.1) for rough data has been the subject of recent studies. In particular, detailed studies of (1.1) have revealed diverse phenomena of the associated data-to-solution map leading to ramified notions of ill-posedness and well-posedness. It is known that:

- The data-to-solution map $H^{s} \ni u_{0} \longmapsto u(t) \in H^{s}$ (for some $t \neq 0$ ) is well-defined and analytic provided $s \geq 0$ [36] [2].

- Uniform continuity of the data-to-solution map from $H^{s}$ to $H^{s}$ fails for $s<0$ [19, 8, 14. Moreover, when $s<0$, the data-to-solution map is discontinuous from $H^{s}(\mathbb{T})$ even to the space of distributions $\left(C^{\infty}(\mathbb{T})\right)^{*}[13,25]$.

- The data-to-solution map is unbounded from $H^{s}(\mathbb{R})$ to $H^{s}(\mathbb{R})$ provided $s<-\frac{1}{2}$. For example, the norm inflation phenomena identified in [14] shows there exist initial data arbitrarily small in $H^{s}(\mathbb{R})$ which evolve into solutions which are arbitrarily large in $H^{s}(\mathbb{R})$ in an arbitrarily short time.

- The data-to-solution map is bounded 2 from $H^{s}(\mathbb{R})$ to $H^{s}(\mathbb{R})$ provided $-\frac{1}{6} \leq s<0$ [21. Moreover, there exist weak solutions associated to every $u_{0} \in H^{s}(\mathbb{R})$ in this range. These weak solutions are not known to be unique.

It is unknown whether well-posedness with merely continuous dependence upon the initial data for (1.1) holds true in $H^{s}$ for $s \geq-\frac{1}{2}$. In contrast to these negative results, this paper establishes positive results on subsets of $H^{s}(\mathbb{T})$ for certain $s<0$ which are full with respect to natural Gaussian measures.

1.2. Invariant Gibbs Measures. Inspired by [23] and following an approach from [40, Bourgain [3] constructed the Gibbs measure for 3 (1.1) and established its invariance under the (1.1) flow. Sufficiently regular solutions of (1.1) satisfy mass conservation

$$
\|u(t)\|_{L^{2}(\mathbb{T})}=\left\|u_{0}\right\|_{L^{2}(\mathbb{T})},
$$

and Hamiltonian conservation

$$
H[u(t)]=\int_{\mathbb{T}} \frac{1}{2}\left|u_{x}(t)\right|^{2} \pm \frac{1}{4}|u(t)|^{4} d x=H\left[u_{0}\right] .
$$

\footnotetext{
${ }^{1}$ We actually consider the Wick ordered version (1.11) instead of (1.1) below.

${ }^{2}$ M. Christ (with J. Holmer and D. Tataru) announced similar results on $\mathbb{T}$ in April 2009 at IHP in Paris.

${ }^{3}$ In fact, the construction and invariance of the Gibbs measure is proved for a family of (sub-)quintic NLS equations containing (1.1) in [3].
} 
By the Hamiltonian structure of the equation, the Gibbs measure

$$
\text { "d } d \mu=e^{-H[u]} \prod_{x \in \mathbb{T}} d u(x) "
$$

is formally invariant. The Gibbs measure is rewritten as a weighted Wiener measure

$$
d \mu=Z^{-1} e^{\mp \frac{1}{4} \int|u|^{4} d x} d \rho
$$

where

$$
d \rho=Z_{0}^{-1} e^{-\frac{1}{2} \int\left|u_{x}\right|^{2} d x} \prod_{x \in \mathbb{T}} d u(x)
$$

is the Wiener measure on $\mathbb{T}$.

The construction of the Gibbs measure proceeds by showing that the density $e^{\mp \frac{1}{4} \int|u|^{4} d x}$ is in $L^{1}(d \rho)$. Expressed in terms of Fourier coefficients, the Wiener measure describes a Gaussian distribution for each $|n| \widehat{u}(n)$. Thus, a typical element in the support of the Wiener measure may be represented

$$
u=u^{\omega}=\sum_{n \in \mathbb{Z}} \frac{g_{n}(\omega)}{|n|} e^{i n x}
$$

where the $\left\{g_{n}\right\}_{n \in \mathbb{Z}}$ are independent standard complex valued Gaussian random variables on some probability space $(\Omega, \mathcal{F}, \mathbb{P})$. Almost surely in $\omega$, the series (1.7) defines a function $u^{\omega} \in H^{\frac{1}{2}-}(\mathbb{T})$. Thus, $\int|u|^{4} d x$ is well-defined and the density $e^{\mp \frac{1}{4} \int|u|^{4} d x}$ may be shown 5 be in $L^{1}(\omega)$.

The invariance of the Gibbs measure is established by studying a sequence of finite dimensional approximations obtained by Dirichlet-projecting the dynamics of (1.1) onto finitely many modes using the fact that the (1.1) evolution is well-defined on the support of the Wiener measure. Recall that the evolution for (1.1) is well-defined for all $u_{0} \in L^{2}(\mathbb{T})$ so it is certainly well-defined on the support of the Gibbs measure living in $H^{\frac{1}{2}-}(\mathbb{T})$.

The questions of existence and invariance of the Gibbs measure associated to (1.1) (in fact, associated to the Wick ordered version (1.11) ) posed on the two-dimensional torus $\mathbb{T}^{2}$ were investigated in [5]. In the two-dimensional case, the representation (1.7) almost surely in $\omega$ defines a distribution in $H^{0-}\left(\mathbb{T}^{2}\right)$ but not in $L^{2}\left(\mathbb{T}^{2}\right)$. More precisely, $u$ defined in (1.7) is almost surely in $B_{2, \infty}^{0}\left(\mathbb{T}^{2}\right) \backslash L^{2}\left(\mathbb{T}^{2}\right)$. Since the data-to-solution map is not welldefined on even $L^{2}\left(\mathbb{T}^{2}\right)$, the issue of well-defined dynamics on the support of the Gibbs measure is not at all obvious. Nonetheless, Bourgain [5] established a well-defined local-intime dynamics on the support of the Wiener measure. In the defocusing case, he proved global well-posedness almost surely on the support, exploiting the invariance of the (finite dimensional) Gibbs measure.

1.3. Almost Sure Local Well-Posedness. Consider the canonical Gaussian measure on $H^{\alpha}(\mathbb{T})$ :

$$
d \widetilde{\rho}_{\alpha}=\widetilde{Z}_{\alpha}^{-1} e^{-\frac{1}{2} \int\left|D^{\alpha} u\right|^{2} d x} \prod_{x \in \mathbb{T}} d u(x)
$$

\footnotetext{
${ }^{4}$ There is an issue regarding the zero Fourier mode which the reader is invited to ignore. The Wiener measure will soon be adjusted using the conserved $L^{2}$ norm into another formally invariant Gaussian measure which avoids the $n=0$ issue.

${ }^{5}$ In the defocusing case, this step is clear. The focusing case requires a more delicate analysis exploiting an (invariant) $L^{2}(\mathbb{T})$ size cutoff (See 23 and $[3]$ ).
} 
where $D=\sqrt{-\partial_{x}^{2}}$. The Gaussian measure $d \rho_{\alpha}$ corresponds to a collection of Gaussian distributions of $\left\{|n|^{\alpha} \widehat{u}(n)\right\}_{n \in \mathbb{Z}}$, so a typical element in the support may be represented $\sqrt{6}$ as a random Fourier series

$$
u=u^{\omega}=\sum_{n \in \mathbb{Z}} \frac{g_{n}(\omega)}{|n|^{\alpha}} e^{i n x} .
$$

This series almost surely in $\omega$ defines a function in $H^{\alpha-\frac{1}{2}-}(\mathbb{T})$ but not in $H^{\alpha-\frac{1}{2}}(\mathbb{T})$. Note that $u_{0}^{\omega}$ in (1.9) can also be expressed as $u_{0}^{\omega}=\sum \widetilde{g}_{n} e_{n}$ where $e_{n}$ is another orthonormal basis in $H^{\alpha}(\mathbb{T})$ and $\left\{\widetilde{g}_{n}\right\}$ is another family of independent standard complex-valued Gaussian random variables. In this respect, the Gaussian measure $\widetilde{\rho}_{\alpha}$ is canonical. See [22] for discussions on the Gaussian measures on Banach spaces. Also, see [41].

Since $\|u(t)\|_{L^{2}}=\left\|u_{0}\right\|_{L^{2}}$ under the flow of (1.1), we formally expect the Gaussian measure on $L^{2}(\mathbb{T})$

$$
d \rho_{0}=Z_{0}^{-1} e^{-\frac{1}{2} \int|u|^{2} d x} \prod_{x \in \mathbb{T}} d u(x)
$$

to be invariant in view of the Hamiltonian structure of (1.1). This measure $\rho_{0}$ is the white noise on the distributions on $\mathbb{T}$ and is supported on $H^{-\frac{1}{2}-}(\mathbb{T}) \backslash H^{-\frac{1}{2}}(\mathbb{T})$, i.e. in the scaling critical/supercritical regime for (1.1). It was shown in [31] that the white noise $\rho_{0}$ is a weak limit of the invariant measures under the flow of (1.1). However, this result does not establish the invariance of the white noise $\rho_{0}$ since the flow is not well-defined on its support. (See Remark 1.3.) Invariance of white noise has recently been established for the $\mathrm{KdV}$ equation on $\mathbb{T}[34,28,31]$. See [29] for a summary of these results.

If we define $v(t)=e^{i \gamma t} u(t)$, with $\gamma \in \mathbb{R}$, where $u$ solves (1.1), then $v$ satisfies $i \partial_{t} v-v_{x x} \pm$ $|v|^{2} v+\gamma v=0$. Recall that $f|u|^{2} d x:=\frac{1}{2 \pi} \int|u|^{2} d x$ is conserved under the flow of (1.1) for $u_{0} \in L^{2}(\mathbb{T})$. Hence, by letting $\gamma=\mp 2 f|u|^{2} d x$, (1.1) is equivalent to

$$
\left\{\begin{array}{l}
i u_{t}-u_{x x} \pm\left(u|u|^{2}-2 u f|u|^{2} d x\right)=0 \\
\left.u\right|_{t=0}=u_{0}
\end{array}\right.
$$

at least for $u_{0} \in L^{2}(\mathbb{T})$. However, for $u_{0} \notin L^{2}(\mathbb{T})$, we can't freely convert solutions of (1.11) into solutions of (1.1). Bourgain [5] refers to (1.11) as the Wick ordered cubic NLS since it may also be obtained from the Wick ordered Hamiltonian.

In the following, we choose to study (1.11) instead of (1.1) for $u_{0} \notin L^{2}(\mathbb{T})$. (See Remark 1.6.) In particular, we consider $u_{0}$ of the form (slightly adjusted compared with (1.9))

$$
u_{0}=u_{0}^{\omega}=\sum_{n \in \mathbb{Z}} \frac{g_{n}(\omega)}{\sqrt{1+|n|^{2 \alpha}}} e^{i n x}
$$

which can be regarded as a typical element in the support of the Gaussian measure

$$
d \rho_{\alpha}=Z_{\alpha}^{-1} \exp \left(-\frac{1}{2} \int|u|^{2} d x-\frac{1}{2} \int\left|D^{\alpha} u\right|^{2} d x\right) \prod_{x \in \mathbb{T}} d u(x) .
$$

By shifting the Laplacian as in [3, 5, i.e. replacing $-u_{x x}$ by $-u_{x x}+u$ in (1.1) or (1.11), we can also regard $u_{0}$ of the form (1.12) as the functions in the support of the Gaussian measure $\widetilde{\rho}_{\alpha}$ defined in (1.8). (Strictly speaking, one needs to replace the denominator in (1.12) by $\left(1+|n|^{2}\right)^{\frac{\alpha}{2}}$ in this case.) Note that $u_{0}^{\omega}$ in (1.12) is in $\bigcap_{s<\alpha-\frac{1}{2}} H^{s} \backslash H^{\alpha-\frac{1}{2}}$. In view

\footnotetext{
${ }^{6}$ The issue with the zero mode should be ignored; see (1.13) below.
} 
of Bourgain's global well-posedness (GWP) result in $L^{2}(\mathbb{T})$ in [2], we assume that $\alpha \leq \frac{1}{2}$ in the following so that $u_{0}^{\omega}$ lies strictly in the negative Sobolev spaces, almost surely in $\omega$.

In establishing local well-posedness, we follow the argument by Bourgain [5]. First, write (1.11) as an integral equation as in (1.14).

$$
u(t)=\Gamma u(t):=S(t) u_{0} \pm i \int_{0}^{t} S\left(t-t^{\prime}\right) \mathcal{N}(u)\left(t^{\prime}\right) d t^{\prime}
$$

where $S(t)=e^{-i \partial_{x}^{2} t}, u_{0}$ is as in (1.12), and

$$
\mathcal{N}(u):=u|u|^{2}-2 u f|u|^{2} .
$$

Note that $S(t) u_{0}$ has the same regularity as $u_{0}$ for each fixed $t \in \mathbb{R}$. i.e. $S(t) u_{0}^{\omega} \in$ $H^{\alpha-\frac{1}{2}-}(\mathbb{T}) \backslash H^{\alpha-\frac{1}{2}}(\mathbb{T})$ a.s. Hence, $S(t) u_{0}$ is strictly in the negative Sobolev space for $\alpha \leq \frac{1}{2}$ a.s.

However, it turns out that the nonlinear part $\int_{0}^{t} S\left(t-t^{\prime}\right) \mathcal{N}(u)\left(t^{\prime}\right) d t^{\prime}$ lies almost surely in a smoother space $L^{2}(\mathbb{T})$ even for $\alpha \leq \frac{1}{2}$. (Also, see [5], [10].) We indeed show that for each small $\delta>0$ there exists $\Omega_{\delta}$ with complemental measure $<e^{-\frac{1}{\delta^{c}}}$ such that $\Gamma$ defined in (1.14) is a contraction on $S(t) u_{0}^{\omega}+B$ for $\omega \in \Omega_{\delta}$ on the time interval $[0, \delta]$, where $B$ denotes the ball of radius 1 in the Bourgain space $X^{s, \frac{1}{2}+, \delta}$ for some $s \geq 0$. (See (2.1) and (2.2) for the definition of $X^{s, \frac{1}{2}+, \delta}$.)

The following theorem states almost sure local well-posedness for each $\alpha \in\left(\frac{1}{6}, \frac{1}{2}\right]$.

Theorem 1. Let $\alpha \in\left(\max \left(\frac{s}{3}+\frac{1}{6}, s\right), \frac{1}{2}\right]$ with $s \in\left[0, \frac{1}{2}\right]$. Then, the periodic (Wick ordered) cubic NLS (1.11) is locally well-posed almost surely in $H^{\alpha-\frac{1}{2}-}(\mathbb{T})$. More precisely, there exist $c>0$ such that for each $\delta \ll 1$, there exists a set $\Omega_{\delta} \in \mathcal{F}$ with the following properties:

(i) The complemental measure of $\Omega_{\delta}$ is small. More precisely, we have

$$
\mathbb{P}\left(\Omega_{\delta}^{c}\right)=\rho_{\alpha} \circ u_{0}\left(\Omega_{\delta}^{c}\right)<e^{-\frac{1}{\delta^{c}}},
$$

where $\rho_{\alpha}$ is the Gaussian probability measure on $H^{\alpha-\frac{1}{2}-}(\mathbb{T})$ defined in (1.13) and $u_{0}$ is viewed as a map $u_{0}: \Omega \rightarrow H^{\alpha-\frac{1}{2}-}(\mathbb{T})$.

(ii) For each $\omega \in \Omega_{\delta}$, there exists a (unique) solution $u$ of (1.11) in

$$
e^{-i \partial_{x}^{2} t} u_{0}+C\left([-\delta, \delta] ; H^{s}(\mathbb{T})\right) \subset C\left([-\delta, \delta] ; H^{\alpha-\frac{1}{2}-}(\mathbb{T})\right)
$$

with the initial condition $u_{0}^{\omega}$ given by (1.12). Here, the uniqueness holds only in the ball centered at $e^{-i \partial_{x}^{2} t} u_{0}$ of radius 1 in $X^{s, \frac{1}{2}+, \delta}$.

In particular, we have almost sure local well-posedness with respect to the Gaussian measure (1.13) supported in $H^{\sigma}(\mathbb{T})$ for each $\sigma>-\frac{1}{3}$.

We prove Theorem 1 in Section 4 by a combination of deterministic multilinear estimates (e.g. Lemma 3.3) and probabilistic estimates on the linear solution with random initial data (Lemmata 3.4, 3.5, and 3.6.)

1.4. Almost Sure Global Well-Posedness. We continue our study on the periodic cubic NLS (1.1) with random initial data in the negative Sobolev spaces. In the second part of this paper, we study global well-posedness of (1.1) with initial data of the form (1.12). In particular, we establish almost sure global well-posedness of (1.1) with respect to the Gaussian measure $\rho_{\alpha}$ in (1.13) for certain values of $\alpha \leq \frac{1}{2}$. 
So far, there is basically only one method known for proving almost sure global wellposedness of PDEs with random initial data of type (1.12). In [3], Bourgain proved the invariance of the Gibbs measures for NLS. In dealing with the super-cubic nonlinearity, (where only the local well-posedness result was available), he used a probabilistic argument and the approximating finite dimensional ODEs (with the invariant finite dimensional Gibbs measures) to extend the local solutions to global ones almost surely on the statistical ensemble and showed the invariance of the Gibbs measures. We point out that this method can be applied in a general setting, provided that local well-posedness is obtained with a "good" estimate on the solutions (e.g. via the fixed point argument) and that we have a formally invariant measure such as the Gibbs measure or the white noise (where the leading term corresponds to (1.13) for $\alpha=1$ and $\alpha=0$.) See Bourgain [4, 5, Burq-Tzvetkov [9, 11, Oh [26, 27, 29], and Tzvetkov [37, 38.

From Theorem 1, we have local solutions in the support of the Gaussian measure $\rho_{\alpha}$ in (1.13) for $\alpha \in\left(\frac{1}{6}, \frac{1}{2}\right]$, which we would like to extend globally in time. Since the values of $\alpha$ is strictly between 0 and 1 , the initial condition $u_{0}$ in (1.12) is not in the support of an invariant measure for (1.11) i.e. $\rho_{\alpha}$ in (1.13) does not correspond to (the quadratic part of) the Gibbs measure or the white noise. Therefore, Bourgain's probabilistic argument [3] is not applicable here.

The crucial point in the local theory is the fact that the nonlinear part is almost surely smoother than the initial data. This observation led us to consider Bourgain's high-low method [6] for establishing global well-posedness, since this kind of nonlinear smoothing is the crucial ingredient for the method. Moreover, as you see below, the implementation of the high-low method naturally lets us apply our probabilistic local theory iteratively since the data for the difference equations with high frequency initial data have random Fourier coefficients (with the same distribution) at each step.

In the following, we briefly sketch the iteration scheme for global well-posedness. Let $s=\alpha-\frac{1}{2}-$ with $\alpha \leq \frac{1}{2}$. i.e. $s<07$

By the large deviation estimate, we have

$$
\mathbb{P}\left(\left\|u_{0}(\omega)\right\|_{H^{s}} \geq K\right) \leq e^{-c K^{2}} .
$$

In the following, we restrict ourselves on $\Omega_{K}=\left\{\omega \in \Omega:\left\|u_{0}(\omega)\right\|_{H^{s}} \leq K\right\}$. By writing $u_{0}=\phi_{0}+\psi_{0}$, where $\phi_{0}:=\mathbb{P}_{\leq N} u_{0}=\sum_{|n| \leq N} \widehat{u}_{0}(n) e^{i n x}$, the low-frequency part $\phi_{0}$ is in $L^{2}(\mathbb{T})$, and it satisfies

$$
\left\|\phi_{0}\right\|_{L^{2}} \leq N^{-s}\left\|\phi_{0}\right\|_{H^{s}} \leq N^{-s} K
$$

Let $u^{1}$ denote the solution of (1.11) with the initial data $\phi_{0}$ on some time interval $[0, \delta]$, where $\delta$ is the time of local existence, i.e. $\delta=\delta\left(N^{-s} K\right) \lesssim \delta\left(\left\|\phi_{0}\right\|_{L^{2}}\right)$. Then, we have

$$
\left\{\begin{array}{l}
i \partial_{t} u^{1}-\partial_{x}^{2} u^{1} \pm \mathcal{N}\left(u^{1}\right)=0 \\
\left.u^{1}\right|_{t=0}=\phi_{0}
\end{array}\right.
$$

From the $L^{2}$ well-posedness theory of Bourgain [2], (1.16) is globally well-posed with the $L^{2}$-conservation: $\left\|u^{1}(t)\right\|_{L^{2}}=\left\|\phi_{0}\right\|_{L^{2}} \lesssim N^{-s} K$ for any $t \in \mathbb{R}$. Moreover, from the local theory, we have

$$
\left\|u^{1}\right\|_{X^{0, \frac{1}{2}+}[0, \delta]} \lesssim\left\|\phi_{0}\right\|_{L^{2}} \leq N^{-s} K
$$

\footnotetext{
${ }^{7}$ In the global theory, we use $s=\alpha-\frac{1}{2}-<0$ to denote the regularity of the initial data below $L^{2}$.
} 
Now, let $v^{1}$ be a solution of the following difference equation on $[0, \delta]$ :

$$
\left\{\begin{array}{l}
i \partial_{t} v^{1}-\partial_{x}^{2} v^{1} \pm\left(\mathcal{N}\left(u^{1}+v^{1}\right)-\mathcal{N}\left(u^{1}\right)\right)=0 \\
\left.v^{1}\right|_{t=0}=\psi_{0}=\sum_{|n|>N} \frac{g_{n}(\omega)}{\sqrt{1+|n|^{2 \alpha}}} e^{i n x}
\end{array}\right.
$$

i.e. we have $u(t)=u^{1}(t)+v^{1}(t)$ as long as the solution $v^{1}$ of (1.18) exists. Note that $\psi_{0}$ has Gaussian-randomized Fourier coefficients. Hence, we can use our probabilistic local theory (as in Theorem 1) to study (1.18).

Suppose that, by our probabilistic local theory, we can show that (1.18) is locally wellposed on the time interval $[0, \delta]$ except on a set of measure $e^{-\frac{1}{\delta^{c}}}$. We have $v^{1}(t)=S(t) \psi_{0}+$ $w^{1}(t)$, where the nonlinear part $w^{1}(t)$ is smoother and is in $L^{2}(\mathbb{T})$ for all $t \in[0, \delta]$. The appearance of the external function $u^{1}$ in (1.18) with large $X_{[0, \delta]}^{0, \frac{1}{2}+}$-norm, forces us to refine our argument used to prove Theorem 1 to obtain a good estimate on $\left\|w^{1}(t)\right\|_{L^{2}}$.

At time $t=\delta$, we redistribute the data. i.e. write $u(\delta)=\phi_{1}+\psi_{1}$, where $\phi_{1}:=$ $u^{1}(\delta)+w^{1}(\delta)$ and $\psi_{1}:=S(\delta) \psi_{0}$. Let $u^{2}$ denote the solution of (1.11) with the initial data $\phi_{1}$ starting at time $t=\delta$. i.e.

$$
\left\{\begin{array}{l}
i \partial_{t} u^{2}-\partial_{x}^{2} u^{2} \pm \mathcal{N}\left(u^{2}\right)=0 \\
\left.u^{2}\right|_{t=\delta}=\phi_{1}=u^{1}(\delta)+w^{1}(\delta) \in L^{2}(\mathbb{T}) .
\end{array}\right.
$$

Then, (1.19) is globally well-posed. Also, from the local theory, we have

$$
\left\|u^{2}\right\|_{X^{0, \frac{1}{2}+}[\delta, 2 \delta]} \lesssim\left\|\phi_{1}\right\|_{L^{2}} \leq\left\|u^{1}(\delta)\right\|_{L^{2}}+\left\|w^{1}(\delta)\right\|_{L^{2}} \lesssim N^{-s} K+\left\|w^{1}(\delta)\right\|_{L^{2}} \lesssim N^{-s} K
$$

as long as

$$
\left\|w^{1}(\delta)\right\|_{L^{2}} \lesssim N^{-s} K
$$

Now, let $v^{2}$ be the solution of the difference equation on $[\delta, 2 \delta]$ :

$$
\left\{\begin{array}{l}
i \partial_{t} v^{2}-\partial_{x}^{2} v^{2} \pm\left(\mathcal{N}\left(u^{2}+v^{2}\right)-\mathcal{N}\left(u^{2}\right)\right)=0 \\
\left.v^{2}\right|_{t=\delta}=\psi_{1}=\sum_{|n|>N} \frac{g_{n}(\omega) e^{i \delta n} n^{2}}{\sqrt{1+|n|^{2 \alpha}}} e^{i n x}
\end{array}\right.
$$

Once again, $\psi_{1}$ has Gaussian-randomized Fourier coefficients. Since the complex Gaussian is invariant under rotation, we see that $\psi_{1}$ has the same distribution as $\psi_{0} 8$ Hence, we can use our probabilistic local theory to study (1.22).

In this way, we iterate the deterministic local theory to the "low-frequency" part $u^{j}$ and the probabilistic local theory to the "high-frequency" part $v^{j}$ to prove that (1.11) is well-posed on $[0, T]$ for arbitrary $T>0$. For details, see Section 5 ,

Theorem 2. Let $\alpha \in\left(\frac{5}{12}, \frac{1}{2}\right]$. Then, the periodic (Wick ordered) cubic NLS (1.11) is globally well-posed almost surely in $H^{\alpha-\frac{1}{2}-}(\mathbb{T})$. More precisely, for almost every $\omega \in \Omega$ there exists a (unique) solution $u$ of (1.11) in

$$
e^{-i \partial_{x}^{2} t} u_{0}+C\left(\mathbb{R} ; L^{2}(\mathbb{T})\right) \subset C\left(\mathbb{R} ; H^{\alpha-\frac{1}{2}-}(\mathbb{T})\right)
$$

with the initial condition $u_{0}^{\omega}$ given by (1.12). Here, the uniqueness holds in a very mild sense. See Remark 1.2.

\footnotetext{
${ }^{8}$ This can be viewed as invariance of the Gaussian measure $\rho_{\alpha}$ (restricted to the high frequencies) under the linear flow. This is the key ingredient for the global-in-time argument (in the absence of (formally) invariant measures under the nonlinear PDE flow.)
} 
In particular, we have almost sure global well-posedness with respect to the Gaussian measure (1.13) supported in $H^{s}(\mathbb{T})$ for each $s>-\frac{1}{12}$.

1.5. Remarks. We conclude this introduction by stating several important remarks.

Remark 1.1. A linear part of a local-in-time solution constructed in Theorem 1 indeed lies in $C([-\delta, \delta] ; B(\mathbb{T}))$ for any Banach space $B(\mathbb{T}) \supset H^{\alpha}(\mathbb{T})$ such that $\left(H^{\alpha}, B, \rho_{\alpha}\right)$ is an abstract Wiener space. (Roughly speaking, an abstract Wiener space is a Banach space extension $B(\mathbb{T})$ of $H^{\alpha}(\mathbb{T})$, where the Gaussian measure $\rho_{\alpha}$ makes sense as a countable additive probability measure.) In this case, a solution $u$ to (1.11) lies in

$$
u=e^{-i \partial_{x}^{2} t} u_{0}+\left(-i \partial_{t}+\partial_{x}^{2}\right)^{-1} u \in C([-\delta, \delta] ; B(\mathbb{T}))+C\left([-\delta, \delta] ; H^{s}(\mathbb{T})\right)
$$

for some $s \geq 0$ as in Theorem 1. As examples of $B$, we can take the Sobolev spaces $W^{\sigma, p}$ with $\sigma<\alpha-\frac{1}{2}$, the Fourier-Lebesgue spaces $\mathcal{F} L^{\sigma, p}$ with $\sigma<\alpha-\frac{1}{p}$, where $\mathcal{F} L^{\sigma, p}$ is defined via the norm $\|f\|_{\mathcal{F} L^{\sigma, p}}=\left\|\langle n\rangle^{\sigma} \widehat{f}(n)\right\|_{L_{n}^{p}}$, and the Besov spaces $B_{p, \infty}^{\alpha-\frac{1}{2}}$ with $p<\infty$. See Bényi-Oh [1] for regularity of $\rho_{\alpha}$ (and $u_{0}$ in (1.12) ) in different function spaces. In [1], we study the regularity of $\rho_{\alpha}$ for $\alpha=1$ but it can be easily adjusted for any $\alpha$. A similar comment applies to global-in-time solutions constructed in Theorem 2. For global-in-time argument, however, it is important that the large deviation estimate (1.15) still holds for these spaces.

Remark 1.2. In the local theory of Theorem 1, uniqueness holds only in the ball centered at $S(t) u_{0}^{\omega}$ of radius 1 in $X^{s, \frac{1}{2}+, \delta}$ for some $s \geq 0$. Continuous dependence on the initial data holds, in some weak sense, in $H^{s}(\mathbb{T})$ for some $s \geq 0$. (See Subsection 4.1) Also, note that Theorem 1 can not be applied to (1.1), since $u_{0}^{\omega}$ is almost surely not in $L^{2}(\mathbb{T})$.

In the global theory of Theorem 2, the situation is a little more complicated. On the one hand, uniqueness and continuous dependence for "low-frequency" part $u^{j}$ in the $j$ th step hold in $C\left([(j-1) \delta, j \delta], L^{2}(\mathbb{T})\right) \cap X^{0, \frac{1}{2}+}[(j-1) \delta, j \delta]$ as usual. On the other hand, uniqueness for the high-frequency part $v^{j}$ in the $j$ th step holds only in the ball centered at $S(t) \psi_{j-1}$ of small radius in $X^{0, \frac{1}{2}+}[(j-1) \delta, j \delta]$. Also, weak continuous dependence for $v^{j}$ holds in $L^{2}(\mathbb{T})$ in the sense analogous to the local theory in Theorem 1 .

Remark 1.3. Recall that the white noise corresponds to $\alpha=0$ in (1.13) (up to constants). Hence, Theorems 1 and 2 may also be viewed as partial results towards showing wellposedness of (1.11) on the support of the white noise $\rho_{0}$.

Remark 1.4. The periodic cubic NLS (1.1) is known to be ill-posed in $H^{s}(\mathbb{T})$ for $s<0$. See Molinet [25] for the most recent work and the references therein. As for the Wick ordered cubic NLS (1.11), note that $u_{N, a}(x, t)=a e^{i\left(N x+N^{2} t \mp|a|^{2} t\right)}$ solves the Wick ordered cubic NLS (1.11) for $a \in \mathbb{C}$ and $N \in \mathbb{N}$. Hence, by following the argument of Burq-GérardTzvetkov [8], we can show failure of uniform continuity of the solution map of (1.11) below $L^{2}(\mathbb{T})$. Thus, it is nontrivial to construct solutions of (1.11) in the negative Sobolev spaces. Also, see Christ-Colliander-Tao [14].

As mentioned earlier, Molinet [25] showed that (1.1) is not well-posedness below $L^{2}(\mathbb{T})$ by proving the weak discontinuity of the flow map in $L^{2}(\mathbb{T})$. We point out that his argument does not apply to (1.11). Indeed, it is shown in [32 that the solution map to the Wick ordered cubic NLS (1.11) is weakly continuous in $L^{2}(\mathbb{T})$.

Remark 1.5. On the one hand, it is known that $u_{0}^{\omega}$ of the form (1.12) is in $\mathcal{F} L^{s, p}$ almost surely for $s<\alpha-\frac{1}{p}$ and not in the smoother spaces. See [28, 1]. On the other hand, Christ 
[12] constructed local-in-time solutions in $\mathcal{F} L^{0, p}$ for $2<p<\infty$ by the power series method. Also see Grünrock-Herr [16] for the same result via the fixed point argument. Hence, it follows from their result that (1.11) with $u_{0}^{\omega}$ in (1.12) is almost surely locally well-posed for $\alpha>0$, but the solution $u$ lies in $C\left([-\delta, \delta] ; \mathcal{F} L^{0, \frac{1}{\alpha}+}(\mathbb{T})\right)$.

In the following, we first construct local-in-time solutions in $C\left([-\delta, \delta] ; H^{\alpha-\frac{1}{2}-}(\mathbb{T})\right)$ by exhibiting nonlinear smoothing under randomization. Also, see Remark 1.1. In Theorem 2, we extend the local solutions to global ones (in the absence of invariant measures) by exploiting such nonlinear smoothing.

Remark 1.6. In [5], the two dimensional Wick ordered (defocusing) cubic NLS appeared as an equivalent formulation of (the limit of the finite dimensional) Hamiltonian equation, arising from the Wick ordered Hamiltonian. Such renormalization on the nonlinearity was a natural consequence of the Euclidean $\varphi_{2}^{4}$ quantum field theory. In our case, by taking the initial data $u_{0}^{\omega}$ to be of the form (1.12) with $\alpha \leq \frac{1}{2}$, (1.11) also arises as an equivalent formulation of (the limit of the finite dimensional) Hamiltonian equation from the Wick ordered Hamiltonian, (at least for $\alpha>\frac{1}{4}$ ) under Gaussian assumption on solutions. Moreover, such renormalization is needed to obtain the continuous dependence on the initial data [12, 16]. See [32] for more discussion on this issue.

Remark 1.7. In [5], local solutions were constructed via the fixed point argument around the linear solution $z_{1}(t):=S(t) u_{0}$ with probabilistic arguments. Also see Burq-Tzvetkov [10, 11] and Thomann [35] for related arguments. While the basic probabilistic argument (e.g. Lemma 3.5) is similar, the argument in [10, 35] further exploits the properties of the eigenfunctions, and the argument in [5] and this paper exploits more properties of the product of Gaussian random variables via the hypercontractivity of the Ornstein-Uhlenbeck semigroup. (See Lemma 3.6.)

In [30, the second author considered KdV and dispersionless Szegö equation with random initial data. Even with random initial data, an attempt to construct local-in-time solutions by the fixed point argument around the linear solution (below the deterministic threshold) failed for both of these equations. Nonetheless, local-in-time solutions for KdV (below the deterministic threshold) were constructed via the second iteration argument, exploiting randomization of initial data.

This paper is organized as follows. In Section 2, we introduce the basic function spaces and notations. In Section 3, we list some deterministic and probabilistic lemmata. Then, we prove Theorem 1 in Section 4 and Theorem 2 in Section 5.

\section{Notation}

First, recall the Bourgain space $X^{s, b}(\mathbb{T} \times \mathbb{R})$, c.f. [2], whose norm is given by

$$
\|u\|_{X^{s, b}(\mathbb{T} \times \mathbb{R})}=\left\|\langle n\rangle^{s}\left\langle\tau-n^{2}\right\rangle^{b} \widehat{u}(n, \tau)\right\|_{l_{n}^{2} L_{\tau}^{2}}
$$

where $\langle\cdot\rangle=1+|\cdot|$. Recall that $X^{s, b}$ embeds into $C_{t} H_{x}^{s}$ for $b>\frac{1}{2}$. We also define the local-in-time version $X^{s, b, \delta}$ on $\mathbb{T} \times[-\delta, \delta]$, by

$$
\|u\|_{X^{s, b, \delta}}=\inf \left\{\|\widetilde{u}\|_{X^{s, b}(\mathbb{T} \times \mathbb{R})}:\left.\widetilde{u}\right|_{[-\delta, \delta]}=u\right\} .
$$

We also define the local-in-time version $X_{I}^{s, b}=X^{s, b}[a, b]$ on an interval $I=[a, b]$. The local-in-time versions of other function spaces are defined analogously.

For simplicity, we often drop $2 \pi$ in dealing with the Fourier transforms. If a function $f$ is random, we may use the superscript $f^{\omega}$ to show the dependence on $\omega$. 
We use $\eta \in C_{c}^{\infty}(\mathbb{R})$ to denote a smooth cutoff function supported on $[-2,2]$ with $\eta \equiv 1$ on $[-1,1]$ and let $\eta_{\delta}(t)=\eta\left(\delta^{-1} t\right)$, and $\chi=\chi_{[-1,1]}$ to denote the characteristic function of the interval $[-1,1]$ and let $\chi_{\delta}(t)=\chi\left(\delta^{-1} t\right)=\chi_{[-\delta, \delta]}(t)$.

The decreasing rearrangement of dyadic numbers $N_{1}, N_{2}, N_{3}$ will be denoted $N^{1}, N^{2}, N^{3}$, following [5].

We use $c, C$ to denote various constants, usually depending only on $\alpha$ and $s$. If a constant depends on other quantities, we will make it explicit. We use $A \lesssim B$ to denote an estimate of the form $A \leq C B$. Similarly, we use $A \sim B$ to denote $A \lesssim B$ and $B \lesssim A$ and use $A \ll B$ when there is no general constant $C$ such that $B \leq C A$. We also use $a+($ and $a-$ ) to denote $a+\varepsilon$ (and $a-\varepsilon$ ), respectively, for arbitrarily small $\varepsilon \ll 1$.

\section{Deterministic and Probabilistic Lemmata}

3.1. Deterministic Lemmata. First, recall the following algebraic identity related to the cubic NLS:

$$
n^{2}-\left(n_{1}^{2}-n_{2}^{2}+n_{3}^{2}\right)=2\left(n_{2}-n_{1}\right)\left(n_{2}-n_{3}\right)
$$

for $n=n_{1}-n_{2}+n_{3}$. Let $N^{1}, N^{2}, N^{3}$ be the decreasing ordering of $N_{1}, N_{2}, N_{3}$, where $\left|n_{j}\right| \sim N_{j}$, and let $n^{j}$ denote the corresponding frequency.

Next, recall the following number theoretic fact [17]. Given an integer $m$, let $d(m)$ denote the number of divisors of $m$. Then, we have

$$
d(m) \lesssim e^{c \frac{\log m}{\log \log m}}\left(=o\left(m^{\varepsilon}\right) \text { for any } \varepsilon>0 .\right)
$$

From this fact, we obtain the following lemma.

Lemma 3.1. Fix $\mu \in \mathbb{Z}$. Let

$$
S_{\mu}=\left\{\left(n_{1}, n_{2}, n_{3}\right) \in \mathbb{Z}^{3}:\left|n_{j}\right| \sim N_{j}, n_{2} \neq n_{1}, n_{3}, \text { and } 2\left(n_{2}-n_{1}\right)\left(n_{2}-n_{3}\right)=\mu\right\} .
$$

Then, we have

$$
\# S_{\mu} \lesssim\left(N^{1}\right)^{0+} N^{3}
$$

Proof. By assumption, we have $|\mu| \lesssim\left(N^{1}\right)^{2}$. Hence, the number of the divisors of $\mu$ is $o\left(\left(N^{1}\right)^{\varepsilon}\right)$ for any $\varepsilon>0$. Without loss of generality, assume $N^{3} \sim \min \left(\left|n_{2}\right|,\left|n_{3}\right|\right)$.

First, suppose $\left|n_{2}\right| \sim N^{3}$. Fix $n_{2}$. Then, from (3.2), there are at most $o\left(\left(N^{1}\right)^{0+}\right)$ many choices for $d:=n_{2}-n_{1}$. Then, there are at most $o\left(\left(N^{1}\right)^{0+}\right)$ many choices for $n_{1}$ and $n_{3}$ since $n_{1}=n_{2}-d$ and $n_{3}=n_{2}-\frac{\mu}{2 d}$.

Next, suppose $\left|n_{3}\right| \sim N^{3}$. Fix $n_{3}$. Then, from (3.2), there are at most $o\left(\left(N^{1}\right)^{0+}\right)$ many choices for $d:=n_{2}-n_{3}$. Then, there are at most $o\left(\left(N^{1}\right)^{0+}\right)$ many choices for $n_{1}$ and $n_{2}$ since $n_{2}=d+n_{3}$ and $n_{1}=d+n_{3}-\frac{\mu}{2 d}$. Hence, (3.3) holds in both cases.

Recall that by restricting the Bourgain spaces onto a small time interval $[-\delta, \delta]$, we can gain a small power of $\delta$ (at a slight loss of regularity on $\left\langle\tau-n^{2}\right\rangle$.) See [2].

Lemma 3.2. Let $s \in \mathbb{R}$ and $b<\frac{1}{2}$. Then, there exists $C=C(b)>0$ such that we have

$$
\|u\|_{X^{s, b, \delta}} \leq C \delta^{\frac{1}{2}-b-}\|u\|_{X^{s, \frac{1}{2}, \delta}} .
$$

Before presenting the proof, first recall the following fact from [7]. Let $\chi_{\delta}(t):=\chi_{[-\delta, \delta]}(t)$ be the characteristic function of the interval $[-\delta, \delta]$. Then, for $b<\frac{1}{2}$, we have

$$
\left\|\chi_{\delta}(t) u\right\|_{X^{s, b}} \sim\|u\|_{X^{s, b, \delta}}
$$


Indeed, by definition (2.2) of local-in-time $X^{s, b}$, we have $\|u\|_{X^{s, b, \delta}} \leq\left\|\chi_{\delta}(t) u\right\|_{X^{s, b}}$. The inequality in the other direction: $\left\|\chi_{\delta}(t) u\right\|_{X^{s, b}} \leq C(b)\|u\|_{X^{s, b, \delta}}$ follows from the boundedness of multiplication by a sharp cutoff function in $H_{t}^{b}$ for $b<\frac{1}{2}$. Note that the constant $C(b)$ depends only on $b$, in particular independent of $\delta$.

Proof. Let $\widetilde{u}$ be any extension of $u$ onto $\mathbb{R}$, i.e. $\widetilde{u}$ is a function on $\mathbb{R}$ such that $\widetilde{u}=u$ on $[-\delta, \delta]$. Also, let $v=\chi_{\delta}(t) \widetilde{u}$. Then, we have $v=\widetilde{u}=u$ on $[-\delta, \delta]$. Moreover, from (3.5), we have

$$
\|v\|_{X^{s, b}} \sim\|\widetilde{u}\|_{X^{s, b, \delta}}=\|u\|_{X^{s, b, \delta}}
$$

for $b<\frac{1}{2}$.

By interpolation, we have

$$
\|v\|_{X^{s, b}} \lesssim\|v\|_{X^{s, 0}}^{\alpha}\|v\|_{X^{s, \frac{1}{2}-}}^{1-\alpha},
$$

where $\alpha=1-(2+) b \in(0,1)$. Recall $\widehat{\chi_{\delta}}(\tau)=\delta \widehat{\chi}(\delta \tau)$, where $\chi=\chi_{[-1,1]}$. Hence, we have

$$
\left\|\widehat{\chi_{\delta}}\right\|_{L_{\tau}^{q}} \sim \delta^{\frac{q-1}{q}}\|\widehat{\chi}\|_{L_{\tau}^{q}} \lesssim \delta^{\frac{q-1}{q}}
$$

where the last inequality holds for $q>1$. Thus, we can gain a positive power of $\delta$ as long as $q>1$. For fixed $n$, by Young and Hölder inequalities, we have

$$
\begin{aligned}
\|\widehat{v}(n, \cdot)\|_{L_{\tau}^{2}} & =\left\|\widehat{\chi_{\delta}} * \widehat{\widetilde{u}}(n, \cdot)\right\|_{L_{\tau}^{2}} \leq\left\|\widehat{\chi_{\delta}}\right\|_{L_{\tau}^{2-}}\|\widehat{\widetilde{u}}(n, \cdot)\|_{L_{\tau}^{1+}} \\
& \lesssim \delta^{\frac{1}{2}-}\left\|\left\langle\tau-n^{2}\right\rangle^{-\frac{1}{2}}\right\|_{L_{\tau}^{2+}}\left\|\left\langle\tau-n^{2}\right\rangle^{\frac{1}{2}} \widehat{\widetilde{u}}(n, \cdot)\right\|_{L_{\tau}^{2}} \\
& \lesssim \delta^{\frac{1}{2}-}\left\|\left\langle\tau-n^{2}\right\rangle^{\frac{1}{2}} \widehat{\widetilde{u}}(n, \cdot)\right\|_{L_{\tau}^{2}}
\end{aligned}
$$

Hence, for $p>2$, we have

$$
\|v\|_{X^{s, 0}} \lesssim \delta^{\frac{1}{2}-}\|\widetilde{u}\|_{X^{s, \frac{1}{2}}}
$$

Then, from (3.6), (3.7), and (3.9), we have

$$
\|u\|_{X^{s, b, \delta}} \sim\|v\|_{X^{s, b}} \lesssim \delta^{\frac{1}{2}-b-}\|\widetilde{u}\|_{X^{s, \frac{1}{2}}}
$$

for any extension $\widetilde{u}$ such that $\widetilde{u}=u$ on $[-\delta, \delta]$. Therefore, (3.4) follows from the definition (2.2).

Lastly, we present the deterministic multilinear estimates. We use them in High Modulation Case (Subsections 4.3 and 5.4.) Recall the periodic $L^{4}$-Strichartz estimate from [2]:

$$
\|u\|_{L_{x, t}^{4}} \lesssim\|u\|_{X^{0, \frac{3}{8}}} .
$$

Interpolating (13.10) with $\|u\|_{L_{x, t}^{2}}=\|u\|_{X^{0,0}}$, we have

$$
\|u\|_{L_{x, t}^{3+}} \lesssim\|u\|_{X^{0, \frac{1}{4}+}} \text {, and }\|u\|_{L_{x, t}^{2+}} \lesssim\|u\|_{X^{0,0+}} .
$$

Lemma 3.3. Let $u_{j}, j=1,2,3,4$, be functions on $\mathbb{T} \times[-\delta, \delta]$. Then, we have (a)

$$
\int_{-\delta}^{\delta} \int_{\mathbb{T}} u_{1} u_{2} u_{3} u_{4} d x d t \lesssim \prod_{j=1}^{4}\left\|u_{j}\right\|_{X^{0, \frac{3}{8}, \delta}}
$$


(b) With large $p$, we have

$$
\int_{-\delta}^{\delta} \int_{\mathbb{T}} u_{1} u_{2} u_{3} u_{4} d x d t \lesssim \prod_{j=1}^{3}\left\|u_{j}\right\|_{X^{0, \frac{1}{4}+, \delta}}\left\|u_{4}\right\|_{L^{p}(\mathbb{T} \times[-\delta, \delta])} .
$$

(c) With large $p$, we have

$$
\int_{-\delta}^{\delta} \int_{\mathbb{T}} u_{1} u_{2} u_{3} u_{4} d x d t \lesssim \prod_{j=1}^{2}\left\|u_{j}\right\|_{X^{0,0+, \delta}} \prod_{j=3}^{4}\left\|u_{j}\right\|_{L^{p}(\mathbb{T} \times[-\delta, \delta])} .
$$

(d) With large $p$, we have

$$
\int_{-\delta}^{\delta} \int_{\mathbb{T}} u_{1} u_{2} u_{3} u_{4} d x d t \lesssim \prod_{j=1}^{2}\left\|u_{j}\right\|_{X^{0, \frac{3}{8}, \delta}}\left\|u_{3}\right\|_{X^{0,0+, \delta}}\left\|u_{4}\right\|_{L^{p}(\mathbb{T} \times[-\delta, \delta])} .
$$

Recall that (3.12) is the essential multilinear estimate for local well-posedness of the cubic NLS in $L^{2}(\mathbb{T})$ by Bourgain [2].

Proof. Let $\widetilde{u}_{j}$ be an extension of $u_{j}$ onto $\mathbb{R}$. Then, by Hölder inequality and (3.10), we have

$$
\text { LHS of }(\underline{3.12}) \leq \prod_{j=1}^{4}\left\|u_{j}\right\|_{L_{x, t}^{4}(\mathbb{T} \times[-\delta, \delta])} \lesssim \prod_{j=1}^{4}\left\|\widetilde{u}_{j}\right\|_{X^{0, \frac{3}{8}, \delta}} .
$$

Hence, (3.12) follows since (3.16) holds for any extensions $\widetilde{u}_{j}$. The other estimates (3.13), (3.14), and (3.15) follow in a similar manner by Hölder inequality:

$$
\begin{aligned}
& 1=\frac{1}{3+}+\frac{1}{3+}+\frac{1}{3+}+\frac{1}{p} \text { for (3.13), } \\
& 1=\frac{1}{2+}+\frac{1}{2+}+\frac{1}{p}+\frac{1}{p} \text { for (3.14), } \\
& 1=\frac{1}{4}+\frac{1}{4}+\frac{1}{2+}+\frac{1}{p} \quad \text { for (3.15), }
\end{aligned}
$$

with (3.10) and (3.11).

3.2. Probabilistic Lemmata. In this subsection, we present several probabilistic lemmata related to the Gaussian random variables. In the following, $\left\{g_{n}\right\}_{n \in \mathbb{Z}}$ denotes a family of independent standard complex valued Gaussian random variables on a probability space $(\Omega, \mathcal{F}, \mathbb{P})$.

Lemma 3.4. Let $\varepsilon, \beta>0$ and $\delta \ll 1$. Then, we have

$$
\left|g_{n}(\omega)\right| \leq C \delta^{-\frac{\beta}{2}}\langle n\rangle^{\varepsilon}
$$

for all $n \in \mathbb{Z}$ for $\omega$ outside an exceptional set of measure $<e^{-\frac{1}{\delta^{c}}}$.

Proof. Recall from [26] that we have $\mathbb{P}\left(\sup _{n}\langle n\rangle^{-\varepsilon}\left|g_{n}(\omega)\right|>K\right) \leq e^{-c K^{2}}$ for sufficiently large $K>0$. Now, choose $K \sim \delta^{-\frac{\beta}{2}}$.

Lemma 3.5. Let $f^{\omega}(x, t)=\sum c_{n} g_{n}(\omega) e^{i\left(n x+n^{2} t\right)}$. Then, for $p \geq 2$, there exists $\delta_{0}>0$ such that

$$
\mathbb{P}\left(\left\|f^{\omega}\right\|_{L^{p}(\mathbb{T} \times[-\delta, \delta])}>C\left\|c_{n}\right\|_{l_{n}^{2}}\right)<e^{-\frac{1}{\delta^{c}}}
$$

for $\delta \leq \delta_{0}$. 
This lemma is in the spirit of Paley-Zygmund [33]. In particular, it says that the linear solution with random initial data satisfies much better Strichartz estimates (with large probability.) Compare with the deterministic case, where Strichartz estimates hold only for $p \leq 4$ (and for $p \leq 6$ with a slight loss of derivative.)

Proof. By separating the real and imaginary parts, assume that $g_{n}$ is real-valued without loss of generality. From the general Gaussian bound (c.f. Burq-Tzvetkov [11]), there exists $C>0$ such that

$$
\left\|\sum_{n} c_{n} g_{n}(\omega)\right\|_{L^{r}(\Omega)} \leq C \sqrt{r}\left\|c_{n}\right\|_{l_{n}^{2}}
$$

for every $r \geq 2$ and every $\left\{c_{n}\right\}_{n \in \mathbb{Z}} \in l_{n}^{2}$. (This is also immediate from the hypercontractivity property as well. See [39].) By Minkowski integral inequality, we have

$$
\begin{aligned}
\mathbb{E}\left(\left\|f^{\omega}\right\|_{L_{x, t}^{p}(\mathbb{T} \times[-\delta, \delta])}^{r}\right)^{\frac{1}{r}} & \leq\|\| f^{\omega}\left\|_{L^{r}(\Omega)}\right\|_{L_{x, t}^{p}} \lesssim \sqrt{r}\|\| c_{n}\left\|_{l_{n}^{2}}\right\|_{L_{x, t}^{p}(\mathbb{T} \times[-\delta, \delta])} \\
& \lesssim \sqrt{r} \delta^{\frac{1}{p}}\left\|c_{n}\right\|_{l_{n}^{2}}
\end{aligned}
$$

for $r \geq p$. Then, by Chebyshev inequality, we have

$$
\mathbb{P}\left(\left\|f^{\omega}\right\|_{L^{p}(\mathbb{T} \times[-\delta, \delta])}>\lambda\right) \leq C^{r} \lambda^{-r} r^{\frac{r}{2}} \delta^{\frac{r}{p}}\left\|c_{n}\right\|_{l_{n}^{2}}^{r} .
$$

Let $\lambda=C r \delta^{\frac{1}{p}}\left\|c_{n}\right\|_{l_{n}^{2}}$ and $r=\delta^{c}$ with $c=\frac{1}{p}$. Then, we have

$$
\mathbb{P}\left(\left\|f^{\omega}\right\|_{L^{p}(\mathbb{T} \times[-\delta, \delta])}>C\left\|c_{n}\right\|_{l_{n}^{2}}\right) \leq e^{-r \ln \sqrt{r}} \leq e^{-\frac{1}{\delta^{c}}},
$$

for $\delta$ sufficiently small such that $r \geq p$. It follows from the proof that $\delta_{0} \sim e^{-p \ln p}$.

The following lemma follows from the hypercontractivity of the Ornstein-Uhlenbeck semigroup, related to products of Gaussian random variables. See Ledoux-Talagrand [24] and Janson [18]. A nice summary is given by Tzvetkov [39, Sections 3 and 4].

Lemma 3.6. For fixed $n \in \mathbb{Z}$, let

$$
D_{n}=\left\{\left(n_{1}, n_{2}, n_{3}\right) \in \mathbb{Z}^{3}: n=n_{1}-n_{2}+n_{3}, n_{2} \neq n_{1}, n_{2} \neq n_{3}, n_{1} \neq n_{3}\right\} .
$$

Given $\left\{a_{n_{1}, n_{2}, n_{3}}\right\} \in l^{2}\left(D_{n}\right)$, define $F_{n}$ by

$$
F_{n}(\omega):=\sum_{\substack{n=n_{1}-n_{2}+n_{3} \\ n_{2} \neq n_{1}, n_{3} \\ n_{1} \neq n_{3}}} a_{n_{1}, n_{2}, n_{3}} g_{n_{1}}(\omega) \overline{g_{n_{2}}}(\omega) g_{n_{3}}(\omega) .
$$

Then, there exists $c>0$ such that, for $\lambda>0$, we have

$$
\mathbb{P}\left(\left|F_{n}(\omega)\right| \geq \lambda\right) \leq \exp \left(-c\left\|F_{n}\right\|_{L^{2}(\Omega)}^{-\frac{2}{3}} \lambda^{\frac{2}{3}}\right) .
$$

Proof. By Propositions 3.1 and 3.3 in [39], we have

$$
\left\|F_{n}\right\|_{L^{p}(\Omega)} \leq p^{\frac{3}{2}}\left\|F_{n}\right\|_{L^{2}(\Omega)},
$$

for all $2 \leq p<\infty$. Then, (3.18) follows from Lemma 4.5 in [39]. 


\section{LOCAL THEORY}

4.1. Basic Setup. Consider the Duhamel formulation (1.14) of the Wick ordered NLS. As mentioned before, when $\alpha \leq \frac{1}{2}$, the linear part $S(t) u_{0}^{\omega} \notin L^{2}(\mathbb{T})$ almost surely. Nonetheless, we show that the nonlinear part lies in a smoother space $H^{s}(\mathbb{T})$ for some $s \geq 0$. More precisely, we prove that for each small $\delta>0$, there exists $\Omega_{\delta}$ with complemental measure $<e^{-\frac{1}{\delta^{c}}}$ such that $\Gamma$ defined in (1.14) is a contraction on $S(t) u_{0}^{\omega}+B$ for $\omega \in \Omega_{\delta}$, where $B$ denotes the ball of radius 1 in $X^{s, \frac{1}{2}+\delta}$ for some $s \geq 0$. i.e. we construct a contraction centered at the linear solution.

Given $u$ on $\mathbb{T} \times[-\delta, \delta]$, let $\widetilde{u}$ be an extension of $u$ onto $\mathbb{T} \times \mathbb{R}$. By the nonhomogeneous linear estimate [2], [15], we have

$$
\begin{aligned}
\left\|\int_{0}^{t} S\left(t-t^{\prime}\right) \mathcal{N}(u)\left(t^{\prime}\right) d t^{\prime}\right\|_{X^{s, \frac{1}{2}+, \delta}} & \leq\left\|\eta_{\delta}(t) \int_{0}^{t} S\left(t-t^{\prime}\right) \mathcal{N}(\widetilde{u})\left(t^{\prime}\right) d t^{\prime}\right\|_{X^{s, \frac{1}{2}+}} \\
& \lesssim\|\mathcal{N}(\widetilde{u})\|_{X^{s,-\frac{1}{2}+}},
\end{aligned}
$$

where $\eta_{\delta}$ is a smooth cutoff on $[-2 \delta, 2 \delta]$. Then, Theorem 1 follows once we prove

$$
\|\mathcal{N}(\widetilde{u})\|_{X^{s,-\frac{1}{2}+}} \lesssim \delta^{\theta}, \quad \text { for some } \theta>0
$$

for $\omega \in \Omega_{\delta}$ with $\mathbb{P}\left(\Omega_{\delta}^{c}\right)<e^{-\frac{1}{\delta^{c}}}$ (for some extension $\widetilde{u}$ of $u$.) From the embedding $X^{s, \frac{1}{2}+, \delta} \subset$ $C\left([-\delta, \delta]: H^{s}\right)$, it follows that (4.1) and (4.2) imply that the nonlinear part of the solution $u^{\omega}$ is in $C\left([-\delta, \delta]: H^{s}\right)$ with large probability. Now, write $\mathcal{N}(u)$ as follows:

$$
\begin{aligned}
\mathcal{N}(u) & =u|u|^{2}-2 u f|u|^{2} \\
(4.3) & =\sum_{n_{2} \neq n_{1}, n_{3}} \widehat{u}\left(n_{1}\right) \widehat{\widehat{u}\left(n_{2}\right)} \widehat{u}\left(n_{3}\right) e^{i\left(n_{1}-n_{2}+n_{3}\right) x}-\sum_{n} \widehat{u}(n)|\widehat{u}(n)|^{2} e^{i n x}=: \mathcal{N}_{1}(u)-\mathcal{N}_{2}(u) .
\end{aligned}
$$

Here, the condition $n_{2} \neq n_{1}, n_{3}$ in the sum for $\mathcal{N}_{1}(u)$ is a shorthand notation for $n_{2} \neq n_{1}$ and $n_{2} \neq n_{3}$. This shorthand notation is used in the remaining part of the paper.

In the following subsections, we will prove (4.2) by separately estimating the contributions from $\mathcal{N}_{1}(\widetilde{u})$ and $\mathcal{N}_{2}(\widetilde{u})$. In particular, we choose an extension $\widetilde{u}$ in $S(t) u_{0}^{\omega}+X^{s, \frac{1}{2}+}$ of $u \in S(t) u_{0}^{\omega}+B$. i.e. $u=S(t) u_{0}^{\omega}+v$ for some $v$ with $\|v\|_{X^{s, \frac{1}{2}+, \delta}} \leq 1$.

By regarding $\mathcal{N}_{1}$ and $\mathcal{N}_{2}$ as trilinear operators, we write

$$
\begin{aligned}
& \mathcal{N}_{1}\left(u_{1}, u_{2}, u_{3}\right)=\sum_{n_{2} \neq n_{1}, n_{3}} \widehat{u}_{1}\left(n_{1}, t\right){\overline{\widehat{u}_{2}}\left(n_{2}, t\right)}_{\widehat{u}_{3}}\left(n_{3}, t\right) e^{i\left(n_{1}-n_{2}+n_{3}\right) x}, \\
& \mathcal{N}_{2}\left(u_{1}, u_{2}, u_{3}\right)=\sum_{n} \widehat{u}_{1}(n, t) \overline{\widehat{u}_{2}(n, t)} \widehat{u}_{3}(n, t) e^{i n x} .
\end{aligned}
$$

Then, we prove (4.2) by carrying out case-by-case analysis on

$$
\left\|\mathcal{N}_{1}\left(u_{1}, u_{2}, u_{3}\right)\right\|_{X^{s,-\frac{1}{2}+}} \quad \text { and } \quad\left\|\mathcal{N}_{2}\left(u_{1}, u_{2}, u_{3}\right)\right\|_{X^{s,-\frac{1}{2}+}},
$$

where $u_{j}$ is taken to be either of type

(I) linear part: random, less regular

$$
u_{j}(x, t)=\sum_{n} \frac{g_{n}(\omega)}{\sqrt{1+|n|^{2 \alpha}}} e^{i\left(n x+n^{2} t\right)}
$$


(II) nonlinear part: deterministic, smoother

$$
u_{j}=\widetilde{v}_{j}, \text { where } \widetilde{v}_{j} \text { is an extension of } v_{j} \text { with }\left\|v_{j}\right\|_{X^{s, \frac{1}{2}+, \delta}} \leq 1 .
$$

In the following, we may insert the smooth cutoff function $\eta_{\delta}$ supported on $[-2 \delta, 2 \delta]$ (or the sharp cutoff function $\chi_{\delta}$ supported on $[-\delta, \delta]$ ) without stating explicitly. This merely corresponds to taking different extensions, and does not cause any problem since our goal is to prove (4.2) for some extension $\widetilde{u}$ of $u$.

Note that (4.1) and (4.2) imply only the boundedness of the map $\Gamma$ in (1.14) from $S(t) u_{0}^{\omega}+B$ into itself (for $\delta>0$ small). In establishing the contraction property, one needs to consider the difference $\Gamma u_{1}-\Gamma u_{2}$ for $u_{1}, u_{2} \in S(t) u_{0}^{\omega}+B$. We omit details since the computation follows in a similar manner. Lastly, suppose that $u_{0}=u_{0}^{\omega}$ is a good initial condition such that $\Gamma$ is a contraction on $S(t) u_{0}+B$. Let $\widetilde{u}_{0}$ be a function on $\mathbb{T}$ such that $\left\|u_{0}-\widetilde{u}_{0}\right\|_{H^{s}}<\frac{1}{10}$. Denote by $\widetilde{\Gamma}$ the solution map corresponding to the initial condition $\widetilde{u}_{0}$. Then, one can show that $\widetilde{\Gamma}$ is also a contraction on $S(t) u_{0}+B$ for $\delta$ sufficiently small. Moreover, we have

$$
\|u(t)-\widetilde{u}(t)\|_{H^{s}} \leq C\left\|u_{0}-\widetilde{u}_{0}\right\|_{H^{s}}
$$

for $|t| \leq \delta$, where $\widetilde{u}$ is the solution with the initial condition $\widetilde{u}_{0}$. For details, see [2], [5].

4.2. Estimate on $\mathcal{N}_{2}$. In this subsection, we prove the easier part of the estimate (4.2):

$$
\left\|\mathcal{N}_{2}\left(u_{1}, u_{2}, u_{3}\right)\right\|_{X^{s,-\frac{1}{2}+}} \lesssim \delta^{\theta}
$$

for some $\theta>0$, outside an exceptional set of measure $e^{-\frac{1}{\delta^{c}}}$, where $\mathcal{N}_{2}$ is as in (4.5) and $u_{j}$ is either of type (I) or (II). We have

$$
\text { LHS of (4.6) }=\left\|\frac{\langle n\rangle^{s}}{\left\langle\tau-n^{2}\right\rangle^{\frac{1}{2}-}} \int_{\tau=\tau_{1}-\tau_{2}+\tau_{3}} \widehat{u}_{1}\left(n, \tau_{1}\right) \overline{\widehat{u}_{2}\left(n, \tau_{2}\right)} \widehat{u}_{3}\left(n, \tau_{3}\right) d \tau_{1} d \tau_{2}\right\|_{l_{n}^{2} L_{\tau}^{2}} .
$$

In the following, we may replace $u_{j}$ by $\eta_{\delta} u_{j}$ if necessary, where $\eta_{\delta}$ the smooth cutoff function supported on $[-2 \delta, 2 \delta]$.

- Case (a): $u_{j}$ of type (II) $, j=1, \ldots, 3$.

By Hölder inequality with $p$ large $\left(\frac{1}{2}=\frac{1}{2+}+\frac{1}{p}\right)$, we have

$$
\text { (4.7) } \lesssim \sup _{n}\left\|\left\langle\tau-n^{2}\right\rangle^{-\frac{1}{2}+}\right\|_{L_{\tau}^{2+}}\left\|\langle n\rangle^{s} \int_{\tau=\tau_{1}-\tau_{2}+\tau_{3}} \widehat{u}_{1}\left(n, \tau_{1}\right) \overline{\widehat{u}_{2}\left(n, \tau_{2}\right)} \widehat{u}_{3}\left(n, \tau_{3}\right) d \tau_{1} d \tau_{2}\right\|_{l_{n}^{2} L_{\tau}^{p}}
$$

By Young and Hölder inequalities,

$$
\lesssim\left\|\langle n\rangle^{s} \prod_{j=1}^{3}\right\| \widehat{\widetilde{v}}_{j}(n, \tau)\left\|_{L_{\tau}^{\frac{3}{2}}-}\right\|_{l_{n}^{2}} \lesssim\left\|\langle n\rangle^{s} \prod_{j=1}^{3}\right\|\left\langle\tau-n^{2}\right\rangle^{\frac{1}{6}+} \widehat{\widetilde{v}}_{j}(n, \tau)\left\|_{L_{\tau}^{2}}\right\|_{l_{n}^{2}}
$$

By Hölder inequality and $l_{n}^{2} \subset l_{n}^{6}$, we have for $s \geq 0$

$$
\lesssim \prod_{j=1}^{3}\left\|\langle n\rangle^{\frac{s}{3}}\left\langle\tau-n^{2}\right\rangle^{\frac{1}{6}+} \widehat{\widetilde{v}}_{j}(n, \tau)\right\|_{l_{n}^{6} L_{\tau}^{2}} \leq \prod_{j=1}^{3}\left\|\widetilde{v}_{j}\right\|_{X^{\frac{s}{3}, \frac{1}{6}+}},
$$


for any extension $\widetilde{v}_{j}$ of $v_{j}$ with $\left\|v_{j}\right\|_{X^{s, \frac{1}{2}+, \delta}} \leq 1$. Hence, by definition (2.2) and Lemma 3.2, we have

$$
\text { (4.7) } \lesssim \prod_{j=1}^{3}\left\|v_{j}\right\|_{X^{\frac{s}{3}, \frac{1}{6}+, \delta}} \lesssim \delta^{1-} \prod_{j=1}^{3}\left\|v_{j}\right\|_{X^{\frac{s}{3}, \frac{1}{2}+, \delta}} \leq \delta^{1-} .
$$

- Case (b): $u_{j}$ of type (I), $j=1, \ldots, 3$.

By Lemma 3.4, we have $\left|g_{n}(\omega)\right| \leq C \delta^{-\frac{\beta}{2}}\langle n\rangle^{\varepsilon}$ for $\omega$ outside an exceptional set of measure $<e^{-\frac{1}{\delta^{c}}}$. Then, by Hölder inequality with $p$ large $\left(\frac{1}{2}=\frac{1}{2+}+\frac{1}{p}\right)$ and Young's inequality with (3.8),

$$
\begin{aligned}
& \text { (4.7) } \lesssim \sup _{n}\left\|\left\langle\tau-n^{2}\right\rangle^{-\frac{1}{2}+}\right\|_{L_{\tau}^{2+}} \\
& \times\left\|\langle n\rangle^{s-3 \alpha}\left|g_{n}\right|^{3} \int_{\tau=\tau_{1}-\tau_{2}+\tau_{3}} \widehat{\eta}_{\delta}\left(\tau_{1}-n^{2}\right) \overline{\widehat{\eta}_{\delta}\left(\tau_{2}-n^{2}\right)} \widehat{\eta}_{\delta}\left(\tau_{3}-n^{2}\right) d \tau_{1} d \tau_{2}\right\|_{l_{n}^{2} L_{\tau}^{p}} \\
& \lesssim \delta^{1-}\left\|\langle n\rangle^{s-3 \alpha}\left|g_{n}(\omega)\right|^{3}\right\|_{l_{n}^{2}} \lesssim \delta^{1-\frac{3}{2} \beta-}\left\|\langle n\rangle^{s-3 \alpha+3 \varepsilon}\right\|_{l_{n}^{2}} \lesssim \delta^{1-}
\end{aligned}
$$

as long as $2 s-6 \alpha+6 \varepsilon<-1$ or $\alpha>\frac{1}{3} s+\frac{1}{6}$.

- Case (c): Exactly two $u_{j}$ 's of type (I). Say $u_{1}(\mathrm{I}), u_{2}(\mathrm{I})$, and $u_{3}(\mathrm{II})$.

By Hölder inequality with $p$ large, Young's inequality with (3.8), and Lemma 3.4, we have

$$
\begin{aligned}
& \text { (4.7) } \lesssim \sup _{n}\left\|\left\langle\tau-n^{2}\right\rangle^{-\frac{1}{2}+}\right\|_{L_{\tau}^{2+}} \\
& \times\left\|\langle n\rangle^{s-2 \alpha}\left|g_{n}\right|^{2} \int_{\tau=\tau_{1}-\tau_{2}+\tau_{3}} \widehat{\eta}_{\delta}\left(\tau_{1}-n^{2}\right) \overline{\widehat{\eta}_{\delta}\left(\tau_{2}-n^{2}\right)} \widehat{\widetilde{v}}_{3}\left(n, \tau_{3}\right) d \tau_{1} d \tau_{2}\right\|_{l_{n}^{2} L_{\tau}^{p}} \\
& \lesssim \delta^{\frac{1}{2}-}\left(\sup _{n}\langle n\rangle^{-2 \alpha}\left|g_{n}\right|^{2}\right)\left\|\langle n\rangle^{s} \widehat{\widetilde{v}}_{3}(n, \tau)\right\|_{l_{n}^{2} L_{\tau}^{2}} \lesssim \delta^{\frac{1}{2}-\beta-}\left\|\widetilde{v}_{3}\right\|_{X^{s, 0}}
\end{aligned}
$$

for $\alpha>0$ outside an exceptional set of measure $<e^{-\frac{1}{\delta c}}$, where $\widetilde{v}_{3}$ is any extension of $v_{3}$ with $\left\|v_{3}\right\|_{X^{s, \frac{1}{2}+, \delta}} \leq 1$. Hence, by definition (2.2) and Lemma 3.2, we have

$$
\text { (4.7) } \lesssim \delta^{1-\beta-}\left\|v_{3}\right\|_{X^{s, \frac{1}{2}+, \delta}} \leq \delta^{1-} \text {. }
$$

- Case (d): Exactly one $u_{j}$ of type (I). Say $u_{1}(\mathrm{I}), u_{2}(\mathrm{I})$, and $u_{3}(\mathrm{II})$.

By Hölder with $p$ large, Young's inequality with (3.8), and Lemma 3.4, we have

$$
\begin{aligned}
& \text { (4.7) } \lesssim \sup _{n}\left\|\left\langle\tau-n^{2}\right\rangle^{-\frac{1}{2}+}\right\|_{L_{\tau}^{2+}} \\
& \times\left\|\langle n\rangle^{s-\alpha}\left|g_{n}\right| \int_{\tau=\tau_{1}-\tau_{2}+\tau_{3}} \widehat{\eta}_{\delta}\left(\tau_{1}-n^{2}\right){\overline{\widehat{\widetilde{v}}_{2}\left(n, \tau_{2}\right)}}_{\widehat{\widetilde{v}}_{3}}\left(n, \tau_{3}\right) d \tau_{1} d \tau_{2}\right\|_{l_{n}^{2} L_{\tau}^{p}} \\
& \lesssim \delta^{\frac{1}{2}-}\left(\sup _{n}\langle n\rangle^{-s-\alpha}\left|g_{n}\right|\right)\left\|\prod_{j=2}^{3}\right\|\langle n\rangle^{s} \widehat{\widetilde{v}}_{j}(n, \tau)\left\|_{L_{\tau}^{\frac{4}{3}}}\right\|_{l_{n}^{2}}
\end{aligned}
$$

By Hölder inequality in $n\left(\frac{1}{2}=\frac{1}{4}+\frac{1}{4}\right)$ and in $\tau\left(\frac{3}{4}=\frac{1}{2}+\frac{1}{4}\right)$ with $l_{n}^{2} \subset l_{n}^{4}$,

$$
\lesssim \delta^{\frac{1}{2}-\frac{\beta}{2}-} \prod_{j=2}^{3}\left\|\langle n\rangle^{s}\left\langle\tau-n^{2}\right\rangle^{\frac{1}{4}+} \widehat{\widetilde{v}}_{j}(n, \tau)\right\|_{l_{n}^{4} L_{\tau}^{2}} \leq \delta^{\frac{1}{2}-\frac{\beta}{2}-} \prod_{j=2}^{3}\left\|\widetilde{v}_{j}\right\|_{X^{s, \frac{1}{4}+}},
$$


for $\alpha>-s$ outside an exceptional set of measure $<e^{-\frac{1}{\delta^{c}}}$, where $\widetilde{v}_{j}, j=2,3$ are any extensions of $v_{j}$ with $\left\|v_{j}\right\|_{X^{s, \frac{1}{2}+, \delta}} \leq 1$. Hence, by definition (2.2) and Lemma 3.2, we have

$$
\text { (4.7) } \lesssim \delta^{1-\frac{\beta}{2}-} \prod_{j=2}^{3}\left\|v_{j}\right\|_{X^{s, \frac{1}{2}+, \delta}} \lesssim \delta^{1-} \text {. }
$$

Remark 4.1. In this subsection, we carefully used the definition (2.2) of the local-in-time space $X^{s, b, \delta}$. Strictly speaking, such a care must be taken in all the subsequent nonlinear analysis. However, this is a routine work and, for simplicity of presentation, we write estimates directly with $\left\|u_{j}\right\|_{X^{s, b, \delta}}$ in the following, meaning that the same estimates hold with $\left\|\widetilde{u}_{j}\right\|_{X^{s, b}}$ for any extension $\widetilde{u}_{j}$ of $u_{j}$ (and thus we can take the infimum over $\widetilde{u}_{j}$.)

4.3. Estimate on $\mathcal{N}_{1}$ : High Modulation Cases. In the next two subsections, we prove the main part of the estimate (4.2):

$$
\left\|\mathcal{N}_{1}\left(u_{1}, u_{2}, u_{3}\right)\right\|_{X^{s,-\frac{1}{2}+}} \lesssim \delta^{\theta}
$$

for some $\theta>0$, outside an exceptional set of measure $e^{-\frac{1}{\delta^{c}}}$, where $\mathcal{N}_{1}$ is as in (4.4) and $u_{j}$ is either of type (I) or (II).

In (most of) the following 9 we assume that $u_{1}, u_{2}, u_{3}$ are dyadically decomposed with frequencies of size $N_{1}, N_{2}, N_{3}$, respectively. As in [5], let $N^{1}, N^{2}, N^{3}$ be the decreasing ordering of $N_{1}, N_{2}, N_{3}$ and $u^{1}, u^{2}, u^{3}$ be the corresponding $u_{j}$-factors. Also, let $\sigma^{1}, \sigma^{2}, \sigma^{3}$ denote the corresponding $\sigma_{j}:=\left\langle\tau_{j}-n_{j}^{2}\right\rangle$. In the following, we use superscripts to imply that the functions (or variables) are arranged in the decreasing order of the spatial frequencies $N_{1}, N_{2}, N_{3}$.

In the rest of this subsection, we consider basic cases. Using duality, we can estimate (4.8) by

$$
\int_{-\delta}^{\delta} \int_{\mathbb{T}}\left(\left\langle\partial_{x}\right\rangle^{s} u^{1}\right) u^{2} u^{3} \cdot v d x d t
$$

where $\|v\|_{X^{0, \frac{1}{2}-, \delta}} \leq 1$ (with the complex conjugate on an appropriate $u^{j}$.) Note that in (4.9), we implicitly inserted the sharp cutoff function $\chi_{\delta}$ (in one of the factors $u^{j}$.)

- Case (A): $u^{1}$ and $u^{2}$ are of type (II).

Suppose that $u^{3}$ is of type (II). In this case, there is no need of apply dyadic on $u^{1}, u^{2}$, and $u^{3}$. By Lemmata 3.3 (a) and 3.2, we have

$$
(4.9) \lesssim \delta^{\frac{1}{2}-}\left\|u^{1}\right\|_{X^{s, \frac{1}{2}, \delta}}\left\|u^{2}\right\|_{X^{0, \frac{1}{2}, \delta}}\left\|u^{3}\right\|_{X^{0, \frac{1}{2}, \delta}}\|v\|_{X^{0, \frac{1}{2}-, \delta}} \leq \delta^{\frac{1}{2}-}
$$

as long as $s \geq 0$.

Next, suppose that $u^{3}$ is of type (I) i.e. $u^{3}=S(t) u_{0}$. In this case, we do not need to apply dyadic decomposition on $u^{1}$ and $u^{2}$. Namely, for a fixed dyadic block $N^{3}$ for $u^{3}$ of type (I), with a slight abuse of notation, we use $u^{1}$ and $u^{2}$ to denote the sums of $u^{j}$ over the dyadic blocks $N^{j} \geq N^{3}, j=1,2$.

By Lemma 3.3 (b) with $p$ large followed by Lemma 3.5, we have

$$
\begin{aligned}
\text { (4.9) } & \lesssim\left\|u^{1}\right\|_{X^{s, \frac{1}{4}+, \delta}}\left\|u^{2}\right\|_{X^{0, \frac{1}{4}+, \delta}}\left\|u^{3}\right\|_{L^{p}}\|v\|_{X^{0, \frac{1}{4}+, \delta}} \\
& \lesssim\left(N^{3}\right)^{\frac{1}{2}-\alpha+}\left\|u^{1}\right\|_{X^{s, \frac{1}{4}+, \delta}}\left\|u^{2}\right\|_{X^{0, \frac{1}{4}+, \delta}}\|v\|_{X^{0, \frac{1}{4}+, \delta}}
\end{aligned}
$$

\footnotetext{
${ }^{9}$ Basically, we only need to dyadically decompose the function $u_{j}$ of type (I).
} 
outside an exceptional set of measure $<e^{-\frac{1}{\delta^{c}}}$. If $\left\langle\tau_{j}-n_{j}^{2}\right\rangle^{\frac{1}{4}-} \gtrsim\left(N^{3}\right)^{\frac{1}{2}-\alpha+}$ for $u_{j}$ of type (II), or if $\left\langle\tau-n^{2}\right\rangle^{\frac{1}{4}-} \gtrsim\left(N^{3}\right)^{\frac{1}{2}-\alpha+}$, then (4.8) follows with $\theta=\frac{1}{2}-$ in view of Lemma $3.2{ }^{10}$

Hence, it remains to estimate the contribution to (4.9) with a cutoff (on the Fourier side) on the region satisfying

$$
\left\langle\tau-n^{2}\right\rangle \ll\left(N^{3}\right)^{2-4 \alpha+} \text {, and }\left\langle\tau_{j}-n_{j}^{2}\right\rangle \ll\left(N^{3}\right)^{2-4 \alpha+} \text { if } u_{j} \text { of type (II). }
$$

- Case (B): $u^{1}$ of type (II), and $u^{2}$ of type (I).

In this case, we do not need to apply dyadic decomposition on $u^{1}$. Namely, for a fixed dyadic block $N^{2}$ for $u^{2}$ of type (I), we use $u^{1}$ to denote the sum of $u^{1}$ over the dyadic blocks $N^{1} \geq N^{2}$.

First, suppose that $u^{3}$ is of type (II). By Lemma 3.3 (b) with $p$ large followed by Lemma 3.5, we have

$$
\begin{aligned}
\text { (4.9) } & \lesssim\left\|u^{1}\right\|_{X^{s, \frac{1}{4}+, \delta}}\left\|u^{2}\right\|_{L^{p}}\left\|u^{3}\right\|_{X^{0, \frac{1}{4}+, \delta}}\|v\|_{X^{0, \frac{1}{4}+, \delta}} \\
& \lesssim\left(N^{2}\right)^{\frac{1}{2}-\alpha+}\left\|u^{1}\right\|_{X^{s, \frac{1}{4}+, \delta}}\left\|u^{3}\right\|_{X^{0, \frac{1}{4}+, \delta}}\|v\|_{X^{0, \frac{1}{4}+, \delta}}
\end{aligned}
$$

outside an exceptional set of size $<e^{-\frac{1}{\delta^{c}}}$. If $\left\langle\tau_{j}-n_{j}^{2}\right\rangle^{\frac{1}{4}-} \gtrsim\left(N^{2}\right)^{\frac{1}{2}-\alpha+}$ for $u_{j}$ of type (II), or if $\left\langle\tau-n^{2}\right\rangle^{\frac{1}{4}-} \gtrsim\left(N^{2}\right)^{\frac{1}{2}-\alpha+}$, then (4.8) follows with $\theta=\frac{1}{2}-$ in view of Lemma 3.2.

Hence, it remains to estimate the contribution to (4.9) from the region satisfying

$$
\left\langle\tau-n^{2}\right\rangle \ll\left(N^{2}\right)^{2-4 \alpha+} \text {, and }\left\langle\tau_{j}-n_{j}^{2}\right\rangle \ll\left(N^{2}\right)^{2-4 \alpha+} \text { if } u_{j} \text { of type (II) }
$$

in the following.

Next, suppose that $u^{3}$ is of type (I). By Lemma 3.3 (c) with $p$ large followed by Lemma 3.5, we have

$$
\begin{aligned}
\text { (44.9) } & \lesssim\left\|u^{1}\right\|_{X^{s, 0+, \delta}}\left\|u^{2}\right\|_{L^{p}}\left\|u^{3}\right\|_{L^{p}}\|v\|_{X^{0,0+, \delta}} \\
& \lesssim\left(N^{2}\right)^{1-2 \alpha+}\left\|u^{1}\right\|_{X^{s, 0+, \delta}}\|v\|_{X^{0,0+, \delta}} .
\end{aligned}
$$

outside an exceptional set of measure $<e^{-\frac{1}{\delta^{c}}}$. If $\left(\sigma^{1}\right)^{\frac{1}{2}-} \gtrsim\left(N^{2}\right)^{1-2 \alpha+}$ or if $\left\langle\tau-n^{2}\right\rangle^{\frac{1}{2}-} \gtrsim$ $\left(N^{2}\right)^{1-2 \alpha+}$, then (4.8) follows with $\theta=\frac{1}{2}-$ in view of Lemma 3.2. Hence, it remains to estimate the contribution to (4.9) from the region satisfying (4.11) as well.

- Case (C): $u^{1}$ of type (I), and $u^{2}, u^{3}$ of type (II).

Suppose $\left\langle\tau-n^{2}\right\rangle \gg \max \left(\sigma^{2}, \sigma^{3}\right)$. By Lemma 3.3 (d) with $p$ large followed by Lemmata 3.5 and 3.2, we have

$$
\begin{aligned}
\text { (4.9) } & \leq\left(N^{1}\right)^{s}\left\|u^{1}\right\|_{L^{p}}\left\|u^{2}\right\|_{X^{0, \frac{3}{8}, \delta}}\left\|u^{3}\right\|_{X^{0, \frac{3}{8}, \delta}}\|v\|_{X^{0,0+, \delta}} \\
& \lesssim\left(N^{1}\right)^{s+\frac{1}{2}-\alpha+}\left\|u^{2}\right\|_{X^{0, \frac{3}{8}, \delta}}\left\|u^{3}\right\|_{X^{0, \frac{3}{8}, \delta}}\|v\|_{X^{0,0+, \delta}} \\
& \lesssim \delta^{\frac{1}{4}-}\left(N^{1}\right)^{s+\frac{1}{2}-\alpha+}\left\|u^{2}\right\|_{X^{0, \frac{1}{2}, \delta}}\left\|u^{3}\right\|_{X^{0, \frac{1}{2}, \delta}}\|v\|_{X^{0,0+, \delta}}
\end{aligned}
$$

outside an exceptional set of measure $\left\langle e^{-\frac{1}{\delta^{c}}}\right.$. Hence, (4.8) follows as long as $\left\langle\tau-n^{2}\right\rangle \gtrsim$ $\left(N^{1}\right)^{2 s+1-2 \alpha+}$. Similar results hold if $\sigma^{2} \gg \max \left(\sigma^{3},\left\langle\tau-n^{2}\right\rangle\right)$ or $\sigma^{3} \gtrsim \max \left(\sigma^{2},\left\langle\tau-n^{2}\right\rangle\right)$.

Hence, it remains to estimate the contribution to (4.9) from the region satisfying

$$
\left\langle\tau-n^{2}\right\rangle \ll\left(N^{1}\right)^{2 s+1-2 \alpha+} \text {, and }\left\langle\tau_{j}-n_{j}^{2}\right\rangle \ll\left(N^{1}\right)^{2 s+1-2 \alpha+} \text { if } u_{j} \text { of type (II). }
$$

\footnotetext{
${ }^{10}$ This is to say that by inserting a cutoff (on the Fourier side) on the region satisfying $\left\langle\tau_{j}-n_{j}^{2}\right\rangle^{\frac{1}{4}-} \gtrsim$ $\left(N^{3}\right)^{\frac{1}{2}-\alpha+}$ for $u_{j}$ of type (II), or $\left\langle\tau-n^{2}\right\rangle^{\frac{1}{4}-} \gtrsim\left(N^{3}\right)^{\frac{1}{2}-\alpha+}$, we can establish (4.8) with $\theta=\frac{1}{2}-$.
} 
- Case (D): $u^{1}$ of type (I), and either $u^{2}(\mathrm{I}), u^{3}(\mathrm{II})$ or $u^{2}(\mathrm{II}), u^{3}(\mathrm{I})$.

Suppose that $u^{2}$ is of type (I) and that $u^{3}$ is of type (II). Moreover, suppose $\left\langle\tau-n^{2}\right\rangle \gg \sigma^{3}$. By Lemma 3.3 (c) with $p$ large followed by Lemmata 3.5 and 3.2, we have

$$
\begin{aligned}
\text { (4.9) } & \leq\left(N^{1}\right)^{s}\left\|u^{1}\right\|_{L^{p}}\left\|u^{2}\right\|_{L^{p}}\left\|u^{3}\right\|_{X^{0,0+, \delta}}\|v\|_{X^{0,0, \delta}} \\
& \lesssim\left(N^{1}\right)^{s+1-2 \alpha+}\left\|u^{3}\right\|_{X^{0,0+, \delta}}\|v\|_{X^{0,0, \delta}} \\
& \lesssim \delta^{\frac{1}{2}-}\left(N^{1}\right)^{s+1-2 \alpha+}\left\|u^{3}\right\|_{X^{0, \frac{1}{2}, \delta}}\|v\|_{X^{0,0, \delta}}
\end{aligned}
$$

outside an exceptional set of measure $\left\langle e^{-\frac{1}{\delta^{c}}}\right.$. Hence, (4.8) follows as long as $\left\langle\tau-n^{2}\right\rangle \gtrsim$ $\left(N^{1}\right)^{2 s+2-4 \alpha+}$. Similar results hold if $\sigma^{3} \gtrsim\left\langle\tau-n^{2}\right\rangle$, (or $u^{2}$ is of type (II) and $u^{3}$ is of type (I).)

Hence, it remains to estimate the contribution to (4.9) from the region satisfying

$$
\left\langle\tau-n^{2}\right\rangle \ll\left(N^{1}\right)^{2 s+2-4 \alpha+} \text {, and }\left\langle\tau_{j}-n_{j}^{2}\right\rangle \ll\left(N^{1}\right)^{2 s+2-4 \alpha+} \text { if } u_{j} \text { of type (II). }
$$

Summary: Given a function $v(x, t)$, we can write $v$ as

$$
v(x, t)=\int\langle\lambda\rangle^{-\frac{1}{2}-}\left(\sum_{n}\langle n\rangle^{2 s}\langle\lambda\rangle^{1+}\left|\widehat{v}\left(n, n^{2}+\lambda\right)\right|^{2}\right)^{\frac{1}{2}}\left\{e^{i \lambda t} \sum_{n} a_{\lambda}(n) e^{i\left(n x+n^{2} t\right)}\right\} d \lambda
$$

where $a_{\lambda}(n)=\frac{\widehat{v}\left(n, n^{2}+\lambda\right)}{\left(\sum_{m}\langle m\rangle^{2 s}\left|\widehat{v}\left(m, m^{2}+\lambda\right)\right|^{2}\right)^{\frac{1}{2}}}$. Note that $\sum_{n}\langle n\rangle^{2 s}\left|a_{\lambda}(n)\right|^{2}=1$. For $\|v\|_{X^{s, \frac{1}{2}+}} \leq 1$, we have

$$
\int_{|\lambda|<K}\langle\lambda\rangle^{-\frac{1}{2}-}\left(\sum_{n}\langle n\rangle^{2 s}\langle\lambda\rangle^{1+}\left|\widehat{v}\left(n, n^{2}+\lambda\right)\right|^{2}\right)^{\frac{1}{2}} d \lambda \lesssim 1
$$

by Cauchy-Schwarz inequality. See (22) and (23) in [5]. Note that (4.14) is a standard representation for functions in $X^{s, b}$ for $b>\frac{1}{2}$. For example, see Klainerman-Selberg [20].

- Case 1: First, we consider the case if any of $u_{j}$ is of type (II). From Cases (A)-(D), we assume that $\left\langle\tau-n^{2}\right\rangle \ll K=K\left(N^{j}\right)$ for $j=1,2$, or 3 in the following. By Hölder inequality, we have

$$
\begin{aligned}
\left\|\mathcal{N}_{1}\right\|_{X^{s,-\frac{1}{2}+\varepsilon}} & =\left(\sum_{n} \int\langle n\rangle^{2 s} \frac{\left|\widehat{\mathcal{N}}_{1}(n, \tau)\right|^{2}}{\left\langle\tau-n^{2}\right\rangle^{1-2 \varepsilon}} d \tau\right)^{\frac{1}{2}}=\left(\sum_{n} \int\langle n\rangle^{2 s} \frac{\left|\widehat{\mathcal{N}}_{1}\left(n, \lambda+n^{2}\right)\right|^{2}}{\langle\lambda\rangle^{1-2 \varepsilon}} d \lambda\right)^{\frac{1}{2}} \\
& \lesssim K^{\varepsilon}\left\|\langle n\rangle^{s} \widehat{\mathcal{N}}_{1}\left(n, \lambda+n^{2}\right)\right\|_{L_{\lambda}^{\infty} l_{n}^{2}}
\end{aligned}
$$

Then, letting $*=\left\{\left(n_{1}, n_{2}, n_{3}\right) \in \mathbb{Z}^{3}: n=n_{1}-n_{2}+n_{3}, n_{2} \neq n_{1}, n_{3}\right\}$ and $*_{*} *_{n}=\left\{\left(\tau_{1}, \tau_{2}, \tau_{3}\right) \in\right.$ $\left.\mathbb{R}^{3}: \tau=\lambda+n^{2}=\tau_{1}-\tau_{2}+\tau_{3}\right\}$, we have

$$
\text { LHS of (4.8) } \lesssim K^{0+} \sup _{\langle\lambda\rangle \ll K}\left\|\sum_{*}\left\langle n^{1}\right\rangle^{s} \int_{* * n} \prod_{j=1}^{3} \widehat{u}_{j}\left(n_{j}, \tau_{j}\right) d \tau_{1} d \tau_{2}\right\|_{l_{n}^{2}},
$$

where $\widehat{u}_{j}\left(n_{j}, \tau_{j}\right)=\frac{g_{n_{j}}(\omega) \delta\left(\tau_{j}-n_{j}^{2}\right)}{\sqrt{1+\left|n_{j}\right|^{2 \alpha}}}$ or

$$
\widehat{u}_{j}\left(n_{j}, \tau_{j}\right)=\int_{\left\{\left|\lambda_{j}\right|<K\right\}}\left\langle\lambda_{j}\right\rangle^{-\frac{1}{2}-} c_{j}\left(\lambda_{j}\right) a_{\lambda_{j}}\left(n_{j}\right) \delta\left(\tau_{j}-n_{j}^{2}-\lambda_{j}\right) d \lambda_{j}
$$

with $\sum_{n_{j}}\left\langle n_{j}\right\rangle^{2 s}\left|a_{\lambda_{j}}\left(n_{j}\right)\right|^{2} \leq 1$ and $c_{j}\left(\lambda_{j}\right)=\left(\sum_{m_{j}}\left\langle m_{j}\right\rangle^{2 s}\left\langle\lambda_{j}\right\rangle^{1+}\left|\widehat{u}_{j}\left(m_{j}, n_{j}^{2}+\lambda_{j}\right)\right|^{2}\right)^{\frac{1}{2}}$. For $j$ such that $u_{j}$ is of type (II), we can pull the integral in the corresponding $\lambda_{j}$ outside the 
$l_{n}^{2}$-norm via Minkowski integral inequality. Then, for fixed $n, n_{j}, \lambda$, and $\lambda_{j}$, by integrating in $\tau_{1}$ and $\tau_{2}$, we obtain

$$
\int_{* *_{n}} \prod_{j=1}^{3} \delta\left(\tau_{j}-n_{j}^{2}-\widetilde{\lambda}_{j}\right) d \tau_{1} d \tau_{2}= \begin{cases}1, & n^{2}-n_{1}^{2}+n_{2}^{2}-n_{3}^{2}+\lambda-\widetilde{\lambda}_{1}+\widetilde{\lambda}_{2}-\widetilde{\lambda}_{3}=0 \\ 0, & \text { otherwise }\end{cases}
$$

where $\widetilde{\lambda}_{j}=0$ or $\lambda_{j}$, corresponding to type(I) or (II).

For example, consider the case when $u_{1}$ and $u_{2}$ are of type (II) and $u_{3}$ is of type (I). Then, from (4.16), we have 11

$$
\begin{aligned}
& \left\|\sum_{*}\left\langle n^{1}\right\rangle^{s} \int_{* *_{n}} \prod_{j=1}^{3} \widehat{u}_{j}\left(n_{j}, \tau_{j}\right) d \tau_{1} d \tau_{2}\right\|_{l_{n}^{2}} \\
& \quad \lesssim \int \prod_{j=1}^{2} \chi_{\left\{\left\langle\lambda_{j}\right\rangle<K\right\}}\left\langle\lambda_{j}\right\rangle^{-\frac{1}{2}-} c_{j}\left(\lambda_{j}\right)\left\|\sum_{*}\left\langle n^{1}\right\rangle^{s} \prod_{k=1}^{2} a_{\lambda_{k}}\left(n_{k}\right) \frac{g_{n_{3}}(\omega)}{\sqrt{1+\left|n_{3}\right|^{2 \alpha}}}\right\|_{l_{n}^{2}} d \lambda_{1} d \lambda_{2} \\
& \quad \lesssim \sup _{\lambda_{1}, \lambda_{2}}\left\|\sum_{*}\left\langle n^{1}\right\rangle^{s} \prod_{j=1}^{2} a_{\lambda_{j}}\left(n_{j}\right) \frac{g_{n_{3}}(\omega)}{\sqrt{1+\left|n_{3}\right|^{2 \alpha}}}\right\|_{l_{n}^{2}}
\end{aligned}
$$

where the last inequality follows from Cauchy-Schwarz inequality and (4.15). Note that if $u_{j}$ is supported on $[-\delta, \delta]$ in time, then, in view of (the proof of) Lemma 3.2, we can gain $\delta^{\theta}$ for small $\theta>0$ in (4.17) by making the power in $\langle\lambda\rangle^{-\frac{1}{2}-}$ slightly larger (keeping it less than $-\frac{1}{2}$ ) in (4.15).

- Case 2: Next, we consider the case when all $u_{j}$ 's are of type (I). From (3.1), we have

$$
\left|\tau-n^{2}\right|=\left|2\left(n_{2}-n_{1}\right)\left(n_{2}-n_{3}\right)\right| \lesssim\left(N^{1}\right)^{2}
$$

By an argument similar to the proof of Lemma 3.2 , we have

$$
\begin{aligned}
\left\|\mathcal{N}_{1}\right\|_{X^{s,-\frac{1}{2}+\varepsilon, \delta}} & \lesssim \delta^{\theta}\left\|\mathcal{N}_{1}\right\|_{X^{s,-\frac{1}{2}+2 \varepsilon, \delta}} \\
& \leq \delta^{\theta}\left\|\left\langle\tau-n^{2}\right\rangle^{-\frac{1}{2}+2 \varepsilon} \sum_{*}\left\langle n^{1}\right\rangle^{s} \int_{* *_{n}} \prod_{j=1}^{3} \frac{g_{n_{j}} \delta\left(\tau_{j}-n_{j}^{2}\right)}{\sqrt{1+\left|n_{j}\right|^{2 \alpha}}} d \tau_{1} d \tau_{2}\right\|_{l_{n}^{2} L_{\tau}^{2}}
\end{aligned}
$$

for small $\theta>0$. By integrating in $\tau_{1}$ and $\tau_{2}$, we have

$$
\int_{* *_{n}} \prod_{j=1}^{3} \delta\left(\tau_{j}-n_{j}^{2}\right) d \tau_{1} d \tau_{2}=\delta\left(\tau-n_{1}^{2}+n_{2}^{2}-n_{3}^{2}\right)
$$

\footnotetext{
${ }^{11}$ We drop the complex conjugate in the following when it plays no role.
} 
Hence, for fixed $n$, we have

$$
\begin{aligned}
& \left\|\left\langle\tau-n^{2}\right\rangle^{-\frac{1}{2}+2 \varepsilon} \sum_{*}\left\langle n^{1}\right\rangle^{s} \int_{* *_{n}} \prod_{j=1}^{3} \frac{g_{n_{j}} \delta\left(\tau_{j}-n_{j}^{2}\right)}{\sqrt{1+\left|n_{j}\right|^{2 \alpha}}} d \tau_{1} d \tau_{2}\right\|_{L_{\tau}^{2}} \\
& =\left(\int\left\langle\tau-n^{2}\right\rangle^{-1+4 \varepsilon}\left|\sum_{\substack{n=n_{1}-n_{2}+n_{3} \\
n_{2} \neq n_{1}, n_{3}}}\left\langle n^{1}\right\rangle^{s} \prod_{j=1}^{3} \frac{g_{n_{j}}}{\sqrt{1+\left|n_{j}\right|^{2 \alpha}}} \delta\left(\tau-n_{1}^{2}+n_{2}^{2}-n_{3}^{2}\right)\right|^{2} d \tau\right)^{\frac{1}{2}}
\end{aligned}
$$

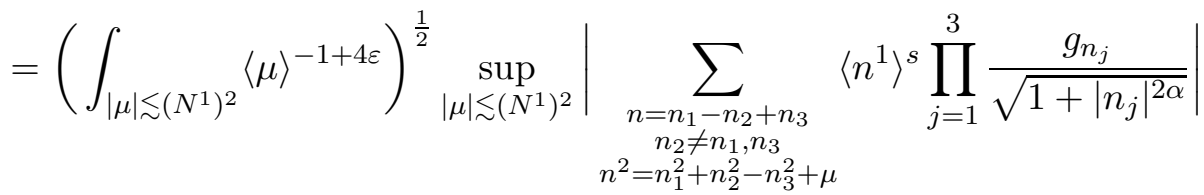

$$
\begin{aligned}
& \lesssim\left(N^{1}\right)^{s+4 \varepsilon} \sup _{|\mu| \lesssim\left(N^{1}\right)^{2}}\left|\sum_{\substack{n=n_{1}-n_{2}+n_{3} \\
n_{2} \neq n_{1}, n_{3} \\
n^{2}=n_{1}^{2}+n_{2}^{2}-n_{3}^{2}+\mu}} \prod_{j=1}^{3} \frac{g_{n_{j}}}{\sqrt{1+\left|n_{j}\right|^{2 \alpha}}}\right| .
\end{aligned}
$$

Then, we can take $l^{2}$-summation in $n$.

Therefore, we can reduce the estimate (4.8) into the following two cases (with $\theta=0+$ ):

- $u^{1}$ is of type (II):

In this case, we do not need to apply dyadic decomposition on $u^{1}$. Namely, for a fixed dyadic block $N^{2}$ for $u^{2}$, with a slight abuse of notation, we use $u^{1}$ to denote the sum of $u^{1}$ over the dyadic blocks $N^{1} \geq N^{2}$.

From (4.10) and (4.11), we can assume that $\sigma_{j} \ll\left(N^{3}\right)^{2-4 \alpha+}$ or $\left(N^{2}\right)^{2-4 \alpha+}$ for $u_{j}$ of type (II). Then, by (4.14) and (4.15), we can bound (4.8) as follows:

$$
\text { (4.8) } \lesssim \delta^{\theta} M\left(N^{2}, N^{3}\right)\left(\sum_{n}\left|\sum_{\substack{n=n_{1}-n_{2}+n_{3} \\ n_{2} \neq n_{1}, n_{3} \\ n^{2}=n_{1}^{2}-n_{2}^{2}+n_{3}^{2}+\mu}} a_{1}\left(n_{1}\right) \overline{a_{2}\left(n_{2}\right)} a_{3}\left(n_{3}\right)\right|^{2}\right)^{\frac{1}{2}} \text {, }
$$

where $\sum_{n}\left|a^{1}(n)\right|^{2} \leq 1, a^{j}(n)=\frac{g_{n}(\omega)}{\sqrt{1+|n|^{2 \alpha}}}$ or $\sum_{|n| \sim N^{j}}\left|a^{j}(n)\right|^{2} \leq\left(N^{j}\right)^{-2 s}$ for $j=2,3$, and

Case (A): $M\left(N^{2}, N^{3}\right)=\left(N^{3}\right)^{0+}$ and $|\mu| \ll\left(N^{3}\right)^{2-4 \alpha+}$

Case (B): $M\left(N^{2}, N^{3}\right)=\left(N^{2}\right)^{0+}$ and $|\mu| \ll\left(N^{2}\right)^{2-4 \alpha+}$.

- $u^{1}$ is of type (I):

From (4.12) and (4.13), we can assume that $\sigma_{j} \ll\left(N^{1}\right)^{2 s+1-2 \alpha+}$ or $\left(N^{1}\right)^{2 s+2-4 \alpha+}$ for $u_{j}$ of type (II). Then, by (4.14) and (4.15), we can bound (4.8) as follows:

$$
\text { (4.8) } \lesssim \delta^{\theta}\left(N^{1}\right)^{s+}\left(\sum_{|n| \lesssim N^{1}}\left|\sum_{\substack{n=n_{1}-n_{2}+n_{3} \\ n_{2} \neq n_{1}, n_{3} \\ n^{2}=n_{1}^{2}-n_{2}^{2}+n_{3}^{2}+\mu}} a_{1}\left(n_{1}\right) \overline{a_{2}\left(n_{2}\right)} a_{3}\left(n_{3}\right)\right|^{2}\right)^{\frac{1}{2}} \text {, }
$$

where $a^{1}(n)=\frac{g_{n}(\omega)}{\sqrt{1+|n|^{2 \alpha}}}, a^{j}(n)=\frac{g_{n}(\omega)}{\sqrt{1+|n|^{2 \alpha}}}$ or $\sum_{|n| \sim N^{j}}\left|a^{j}(n)\right|^{2} \leq\left(N^{j}\right)^{-2 s}$ for $j=2,3$, and

Case $(\mathrm{C}): \quad|\mu| \ll\left(N^{1}\right)^{2 s+1-2 \alpha+}$

Case (D): $\quad|\mu| \ll\left(N^{1}\right)^{2 s+2-4 \alpha+}$

All type (I): $|\mu| \ll\left(N^{1}\right)^{2}$, 
Note that all the spatial frequencies are dyadically decomposed in this case.

Suppose $\left|n_{2}\right|>10\left(\left|n_{1}\right|+\left|n_{3}\right|\right)$. Then, on the one hand, $|\mu| \sim\left|\left(n_{2}-n_{1}\right)\left(n_{2}-n_{3}\right)\right| \sim\left|n_{2}\right|^{2} \sim$ $\left(N^{1}\right)^{2}$ by (3.1). On the other hand, if $u^{1}=u_{2}$ is of type (II), we have $|\mu| \ll\left(N^{2}\right)^{2-4 \alpha+} \ll$ $\left(N^{1}\right)^{2}$ as long as $\alpha>0$. If $u^{1}=u_{2}$ is of type (I), we have $|\mu| \ll\left(N^{1}\right)^{2 s+2-4 \alpha+} \ll\left(N^{1}\right)^{2}$ since $\alpha>\frac{s}{2}$. In both cases, we would have a contradiction. Hence, we can assume that $\left|n_{1}\right| \sim N^{1}$ or $\left|n_{3}\right| \sim N^{1}$. Moreover, by symmetry between $u_{1}$ and $u_{3}$, we assume $\left|n_{1}\right| \sim N^{1}$ in the following.

Lastly, we list all the different cases following [5]. We consider these cases in details in the next subsection.

Case (a): $n_{1}=N^{1}(\Pi), n_{2}=N^{2}(\mathrm{I}), n_{3}=N^{3}\left(\right.$ II) or $n_{2}=N^{3}(\mathrm{I}), n_{3}=N^{2}(\Pi)$

Case (b): $n_{1}=N^{1}(\mathrm{I}), n_{2}=N^{3}(\mathrm{I}), n_{3}=N^{2}(\mathrm{I})$ or $n_{2}=N^{2}(\mathrm{I}), n_{3}=N^{3}(\mathrm{I})$

Case (c): $n_{1}=N^{1}(\mathrm{I}), n_{2}=N^{2}\left(\right.$ II),$n_{3}=N^{3}($ II)

Case (d): $n_{1}=N^{1}(\mathrm{I}), n_{2}=N^{3}($ II) $), n_{3}=N^{2}($ II $)$

Case (e): $n_{1}=N^{1}(\mathrm{II}), n_{2}=N^{2}(\mathrm{I}), n_{3}=N^{3}(\mathrm{I})$

Case (f): $n_{1}=N^{1}\left(\right.$ II) $, n_{2}=N^{3}(\mathrm{I}), n_{3}=N^{2}(\mathrm{I})$

Case (g): $n_{1}=N^{1}(\mathrm{I}), n_{2}=N^{2}(\mathrm{I}), n_{3}=N^{3}(\mathrm{II})$

Case (h): $n_{1}=N^{1}(\mathrm{I}), n_{2}=N^{3}(\mathrm{I}), n_{3}=N^{2}(\mathrm{II})$

Case (i): $n_{1}=N^{1}(\mathrm{I}), n_{2}=N^{2}(\mathrm{II}), n_{3}=N^{3}(\mathrm{I})$

Case (j): $n_{1}=N^{1}(\mathrm{I}), n_{2}=N^{3}(\mathrm{II}), n_{3}=N^{2}(\mathrm{I})$

Case (k): $n_{1}=N^{1}(\mathrm{I}), n_{2}=N^{2}(\mathrm{I}), n_{3}=N^{3}(\mathrm{I})$

Case (l): $n_{1}=N^{1}(\mathrm{I}), n_{2}=N^{3}(\mathrm{I}), n_{3}=N^{2}(\mathrm{I})$

4.4. Estimate on $\mathcal{N}_{1}$ : Low Modulation Cases. For notational simplicity, we use $|n|^{\alpha}$ for $\sqrt{1+|n|^{2 \alpha}}$. We may drop a complex conjugate on $u_{2}$ when it plays no significant role. Now, let

$$
\begin{aligned}
A_{n}=\left\{\left(n_{1}, n_{2}, n_{3}\right) \in \mathbb{Z}^{3}: n=\right. & n_{1}-n_{2}+n_{3},\left|n_{j}\right| \sim N_{j}, j=2,3, \\
& \left.n_{2} \neq n_{1}, n_{3}, \text { and } n^{2}=n_{1}^{2}-n_{2}^{2}+n_{3}^{2}+\mu\right\}
\end{aligned}
$$

and $B_{n}=A_{n} \cap\left\{\left|n_{1}\right| \sim N_{1}\right\}$. Also, from (3.1), we have

$$
\mu=2\left(n_{2}-n_{1}\right)\left(n_{2}-n_{3}\right)=2\left(n-n_{1}\right)\left(n-n_{3}\right) .
$$

- Cases (k), (l): $u_{1}, u_{2}, u_{3}$ of type (I). In this case, we have

$$
\text { (4.20) } \lesssim \delta^{\theta} N_{1}^{s+}\left(\sum_{|n| \lesssim N_{1}} \mid \sum_{B_{n}} \frac{g_{n_{1}}}{\left|n_{1}\right|^{\alpha}} \frac{\overline{g_{n_{2}}}}{\left|n_{2}\right|^{\alpha}} \frac{g_{n_{3}}}{\left.\left|n_{3}\right|^{\alpha}\right|^{2}}\right)^{\frac{1}{2}} \text {. }
$$

First, we consider the contribution from $n_{1} \neq n_{3}$. Let

$$
F_{n}(\omega):=\sum_{C_{n}} \frac{g_{n_{1}}(\omega)}{\left|n_{1}\right|^{\alpha}} \frac{\overline{g_{n_{2}}}(\omega)}{\left|n_{2}\right|^{\alpha}} \frac{g_{n_{3}}(\omega)}{\left|n_{3}\right|^{\alpha}}
$$

where $C_{n}=B_{n} \cap\left\{n_{1} \neq n_{3}\right\}$. Then, applying Lemma 3.6 for with $\lambda=\delta^{-\frac{3}{2} \beta} N_{1}^{\frac{3}{2} \varepsilon}\left\|F_{n}\right\|_{L^{2}(\Omega)}$ with $\varepsilon=0+$, we have

$$
\mathbb{P}\left(\left|F_{n}(\omega)\right| \geq \delta^{-\frac{3}{2} \beta} N_{1}^{\frac{3}{2} \varepsilon}\left\|F_{n}\right\|_{L^{2}(\Omega)}\right) \leq e^{-\frac{c^{\prime} N_{1}^{\varepsilon}}{\delta^{\beta}}} .
$$


By Lemma 3.1, we have

$$
\begin{aligned}
\text { RHS of (4.22) } & \lesssim \delta^{\theta-\frac{3}{2} \beta} N_{1}^{s+\frac{3}{2} \varepsilon+}\left(\sum_{|n| \lesssim N_{1}} \sum_{C_{n}} \frac{1}{\left|n_{1}\right|^{2 \alpha}\left|n_{2}\right|^{2 \alpha}\left|n_{3}\right|^{2 \alpha}}\right)^{\frac{1}{2}} \\
& \lesssim \delta^{\theta-\frac{3}{2} \beta} N_{1}^{s-\alpha+\frac{3}{2} \varepsilon+}\left(N^{2}\right)^{-\alpha}\left(N^{3}\right)^{-\alpha+\frac{1}{2}} \\
& \lesssim \begin{cases}\delta^{\theta-\frac{3}{2} \beta} N_{1}^{s-3 \alpha+\frac{1}{2}+\frac{3}{2} \varepsilon+} \quad \text { for } \alpha \leq \frac{1}{4} \\
\delta^{\theta-\frac{3}{2} \beta} N_{1}^{s-\alpha+\frac{3}{2} \varepsilon+} \quad \text { for } \alpha \geq \frac{1}{4}\end{cases} \\
& \leq \delta^{\theta-\frac{3}{2} \beta} \prod_{j=1}^{3} N_{j}^{0-}, \begin{cases}\text { for } \alpha>\frac{s}{3}+\frac{1}{6} & \text { (with } \left.\alpha \leq \frac{1}{4}\right) \\
\text { for } \alpha>s & \text { (with } \left.\alpha \geq \frac{1}{4}\right)\end{cases}
\end{aligned}
$$

outside an exceptional set of measure

$$
<\sum_{|n| \lesssim N_{1}} e^{-\frac{c^{\prime} N_{1}^{\varepsilon}}{\delta^{\beta}}} \lesssim N_{1} e^{-\frac{c^{\prime} N_{1}^{\varepsilon}}{\delta^{\beta}}} \leq N_{1}^{0-} e^{-\frac{c^{\prime}}{\delta^{\beta}} N_{1}^{\varepsilon}+(1+) \log N_{1}}<N_{1}^{0-} e^{-\frac{1}{\delta^{c}}} .
$$

Note that in this case we need to make sure that the measures of these exceptional sets corresponding to different dyadic blocks are indeed summable and bounded by $e^{-\frac{1}{\delta^{c}}}$. We may not be explicit about this point in other cases. e.g. Cases (A)-(D) in Subsection 4.3. We do not encounter this issue in using Lemma 3.4 since it gives one exceptional set of measure $<e^{-\frac{1}{\delta^{c}}}$ for all the frequencies.

Now, consider the contribution from $n_{1}=n_{3}$. It follows from (4.21) that there is at most one choice of $\left(n_{1}, n_{2}, n_{3}\right)$ for each fixed $n$. Thus, $\sum_{|n| \lesssim N_{1}}\left|\sum_{B_{n}, n_{1}=n_{3}} 1\right|^{2}=$ $\sum_{|n| \lesssim N_{1}} \sum_{B_{n}, n_{1}=n_{3}} 1$. Hence, by Lemmata 3.1 and 3.4, we have

$$
\text { RHS of (4.22) } \lesssim \delta^{\theta-\frac{3}{2} \beta} N_{1}^{s-2 \alpha+2 \varepsilon+} N_{2}^{-\alpha+\frac{1}{2}+\varepsilon} \leq \delta^{\theta-\frac{3}{2} \beta} \prod_{j=1}^{3} N_{j}^{0-}
$$

for $\alpha>\frac{s}{3}+\frac{1}{6}$ outside an exceptional set of measure $<e^{-\frac{1}{\delta^{c}}}$.

- Case (a): (Case (b) can be treated in a similar way by replacing $n_{2}$ and $n_{3}$.)

In this case, we have $\mu=2\left(n_{2}-n_{1}\right)\left(n_{2}-n_{3}\right)=o\left(\left(N_{2}\right)^{2-4 \alpha+}\right)$. This implies that $|n|,\left|n_{1}\right|,\left|n_{3}\right| \lesssim N_{2}^{q}$ for some $q>0$ since $n_{2} \neq n_{1}, n_{3}$. Now, fix $n$. Then, it follows from (3.2) that

$$
\sum_{A_{n}} 1 \lesssim N_{2}^{\varepsilon}
$$

Then, by Lemma 3.4 and Cauchy-Schwarz inequality, we have

$$
(\underline{4.19}) \lesssim \delta^{\theta-\frac{\beta}{2}}\left(N_{2}\right)^{-\alpha+\frac{1}{2} \varepsilon+}\left(\sum_{n}\left(\sum_{A_{n}}\left|a_{1}\left(n_{1}\right)\right|^{2}\left|a_{3}\left(n_{3}\right)\right|^{2}\right)\left(\sum_{A_{n}} 1\right)\right)^{\frac{1}{2}}
$$

By (4.26), we have

$$
\begin{aligned}
& \lesssim \delta^{\theta-\frac{\beta}{2}} N_{2}^{-\alpha+\varepsilon+}\left(\sum_{n} \sum_{A_{n}}\left|a_{1}\left(n_{1}\right)\right|^{2}\left|a_{3}\left(n_{3}\right)\right|^{2}\right)^{\frac{1}{2}} \\
& \lesssim \delta^{\theta-\frac{\beta}{2}} N_{2}^{-\alpha+\varepsilon+} N_{3}^{-s} \leq \delta^{\theta^{\prime}} N_{2}^{0-} N_{3}^{0-}
\end{aligned}
$$


for $\alpha>0$ and $s \geq 0$ outside an exceptional set of measure $<e^{-\frac{1}{\delta^{c}}}$. Note that $\left|n_{3}\right| \lesssim N_{2}^{q}$ is crucial in the last inequality of (4.27) when $s=0, n_{2}=N^{3}$, and $n_{3}=N^{2}$.

In Case (b), we have $\mu=2\left(n_{2}-n_{1}\right)\left(n_{2}-n_{3}\right)=o\left(\left(N_{3}\right)^{2-4 \alpha+}\right)$, which implies that $|n|,\left|n_{1}\right|,\left|n_{2}\right| \lesssim N_{3}^{q}$ for some $q>0$ since $n_{2} \neq n_{1}, n_{3}$. The rest of the argument follows as above by replacing $n_{2}$ and $n_{3}$.

- Case (c): (Case (d) can be treated in a similar way by replacing $n_{2}$ and $n_{3}$.)

Let $b_{2}\left(n_{2}\right)=\left|n_{2}\right|^{s} a_{2}\left(n_{2}\right)$. Then, we have $\sum_{\left|n_{2}\right| N_{2}}\left|b_{2}\left(n_{2}\right)\right|^{2} \lesssim 1$. By Lemma 3.4 and Cauchy-Schwarz inequality on $n_{3}$ in the inner sum,

$$
\text { (4.20) } \lesssim \delta^{\theta-\frac{\beta}{2}} N_{1}^{s-\alpha+\varepsilon+} N_{2}^{-s} N_{3}^{-s}\left(\sum_{|n| \lesssim N^{1}} \sum_{B_{n}}\left|b_{2}\left(n_{2}\right)\right|^{2}\right)^{\frac{1}{2}}
$$

outside an exceptional set of measure $<e^{-\frac{1}{\delta^{c}}}$. For fixed $n_{2}$, it follows from (the proof of) Lemma 3.1 that there are at most $N_{1}^{0+}$ terms in the sum. Hence, we have

$$
(4.20) \lesssim \delta^{\theta-\frac{\beta}{2}} N_{1}^{s-\alpha+\varepsilon+} N_{2}^{-s} N_{3}^{-s} \leq \delta^{\theta^{\prime}} N_{1}^{0-} N_{2}^{0-} N_{3}^{0-}
$$

for $\alpha>s \geq 0$.

- Case (e): (Case (f) is basically the same.)

In this case, we have $|\mu|=\left|2\left(n_{2}-n_{1}\right)\left(n_{2}-n_{3}\right)\right| \ll N_{2}^{2-4 \alpha+}$. Fix $n$. Then, from (3.2), there are at most $d(\mu)=O\left(N_{2}^{0+}\right)$ many choices for $n_{2}$ and $n_{3}$. Then, by Lemma 3.4, Cauchy-Schwarz inequality, and (4.26), we have

$$
\begin{aligned}
\text { (4.19) } & \lesssim \delta^{\theta-\beta} N_{2}^{-\alpha+\varepsilon+} N_{3}^{-\alpha}\left(\sum_{|n| \lesssim N_{2}^{q}}\left(\sum_{A_{n}}\left|a_{1}\left(n_{1}\right)\right|^{2}\right)\left(\sum_{A_{n}} 1\right)\right)^{\frac{1}{2}} \\
& \lesssim \delta^{\theta-\beta} N_{2}^{-\alpha+\frac{3}{2} \varepsilon+} N_{3}^{-\alpha}\left(\sum_{|n| \lesssim N_{2}^{q}} \sum_{A_{n}}\left|a_{1}\left(n_{1}\right)\right|^{2}\right)^{\frac{1}{2}} \\
& \lesssim \delta^{\theta-\beta} N_{2}^{-\alpha+2 \varepsilon+} N_{3}^{-\alpha} \leq \delta^{\theta^{\prime}} N_{2}^{0-} N_{3}^{0-}
\end{aligned}
$$

for $\alpha>0$ outside an exceptional set of measure $<e^{-\frac{1}{\delta c}}$ (with some $q>0$ as in Case (a).) In the above computation, we used

$$
\sum_{|n| \lesssim N_{2}^{q}} \sum_{A_{n}}\left|a_{1}\left(n_{1}\right)\right|^{2} \lesssim N_{2}^{\varepsilon} \sum_{n_{1}}\left|a_{1}\left(n_{1}\right)\right|^{2} \leq N_{2}^{\varepsilon}
$$

by first summing over $n_{2}$ (for fixed $n_{1}$ ) and then over $n_{1}$.

- Case (g): (Cases (h), (i), (j) are basically the same.)

Fix $n$. Then, from (3.2), there are at most $d(\mu)=O\left(N_{1}^{0+}\right)$ many choices for $n_{2}$ and $n_{3}$. Thus, we have $\sum_{A_{n}} 1 \lesssim N_{1}^{\varepsilon}$. Then, by Lemma 3.4 and Cauchy-Schwarz inequality as before, we have

$$
\begin{aligned}
(4.20) & \lesssim \delta^{\theta-\beta} N_{1}^{s-\alpha+\varepsilon+} N_{2}^{-\alpha+\frac{1}{2} \varepsilon}\left(\sum_{|n| \lesssim N_{1}} \sum_{A_{n}}\left|a_{3}\left(n_{3}\right)\right|^{2}\right)^{\frac{1}{2}} \\
& \lesssim \delta^{\theta-\beta} N_{1}^{s-\alpha+\frac{3}{2} \varepsilon+} N_{2}^{-\alpha+\frac{1}{2} \varepsilon} N_{3}^{-s} \leq \delta^{\theta^{\prime}} \prod_{j=1}^{3} N_{j}^{0-}
\end{aligned}
$$

for $\alpha>s \geq 0$ outside an exceptional set of measure $<e^{-\frac{1}{\delta^{c}}}$. 
This completes the proof of Theorem 1 .

\section{Global TheOry}

In this section, we prove almost sure global well-posedness of (1.11).

5.1. Reduction of Theorem 2 to Proposition 5.2. In this subsection, we first prove Theorem 2, assuming the following proposition. Heuristically speaking, this says "almost" almost sure global well-posedness (Proposition 5.1) implies almost sure global wellposedness (Theorem 2, )

Proposition 5.1. Let $\alpha \in\left(\frac{5}{12}, \frac{1}{2}\right]$. Given $T>0$ and $\varepsilon>0$, there exists $\Omega_{T, \varepsilon} \in \mathcal{F}$ with the following properties:

(i) $\mathbb{P}\left(\Omega_{T, \varepsilon}^{c}\right)=\rho_{\alpha} \circ u_{0}\left(\Omega_{T, \varepsilon}^{c}\right)<\varepsilon$, where $\rho_{\alpha}$ is the Gaussian probability measure on $H^{\alpha-\frac{1}{2}-}(\mathbb{T})$ defined in (1.13) and $u_{0}$ is viewed as a map $u_{0}: \Omega \rightarrow H^{\alpha-\frac{1}{2}-}(\mathbb{T})$.

(ii) For each $\omega \in \Omega_{T, \varepsilon}$ there exists a (unique) solution $u$ of (1.11) in

$$
e^{-i \partial_{x}^{2} t} u_{0}+C\left([-T, T] ; L^{2}(\mathbb{T})\right) \subset C\left([-T, T] ; H^{\alpha-\frac{1}{2}-}(\mathbb{T})\right)
$$

with the initial condition $u_{0}^{\omega}$ given by (1.12). Here, the uniqueness holds in a very mild sense. See Remark 1.2.

Proof of Theorem 2. For fixed $\varepsilon>0$, let $T_{j}=2^{j}$ and $\varepsilon_{j}=2^{-j} \varepsilon$. Apply Proposition 5.1 and construct $\Omega_{T_{j}, \varepsilon_{j}}$. Then, let $\Omega_{\varepsilon}=\bigcap_{j=1}^{\infty} \Omega_{T_{j}, \varepsilon_{j}}$. Note that (1.11) is globally well-posed on $\Omega_{\varepsilon}$ with $\mathbb{P}\left(\Omega_{\varepsilon}^{c}\right)<\varepsilon$. Now, let $\widetilde{\Omega}=\bigcup_{\varepsilon>0} \Omega_{\varepsilon}$. Then, (1.11) is globally well-posed on $\widetilde{\Omega}$ and $\mathbb{P}\left(\widetilde{\Omega}^{c}\right)=0$.

Now, we present the proof of Proposition 5.1.

Proof of Proposition 5.1. First, recall the following argument which relates the time of local existence $\delta$ and the size of the initial condition. Consider (1.11). We briefly review the deterministic $L^{2}$-local theory. For $t \in[-\delta, \delta]$, (1.14) is equivalent to

$$
u(t)=S(t) u_{0} \pm i \int_{0}^{t} S\left(t-t^{\prime}\right) \mathcal{N}\left(\chi_{\delta}\left(t^{\prime}\right) u\right)\left(t^{\prime}\right) d t^{\prime} .
$$

By (4.1), we have

$$
\|u\|_{X^{0, \frac{1}{2}+\varepsilon_{1}, \delta}} \leq C_{0}\left\|u_{0}\right\|_{L^{2}}+C_{1}\left\|\mathcal{N}\left(\chi_{\delta} \widetilde{u}\right)\right\|_{X^{0,-\frac{1}{2}+\varepsilon_{1}}}
$$

for any extension $\widetilde{u}$ of $u$. By duality (against $v$ in $X^{0, \frac{1}{2}-\varepsilon_{1}}$ ) with Lemma 3.3 (a) followed by Lemma 3.2 ,

$$
\leq C_{0}\left\|u_{0}\right\|_{L^{2}}+C_{2} \delta^{\frac{1}{2}-\varepsilon_{1}-\varepsilon_{2}}\|\widetilde{u}\|_{X^{0, \frac{1}{2}+\varepsilon_{1}}}^{3}
$$

for some small $\varepsilon_{2}>0$. Hence, we obtain

$$
\|u\|_{X^{0, \frac{1}{2}+, \delta}} \leq C_{0}\left\|u_{0}\right\|_{L^{2}}+C_{2} \delta^{\frac{1}{2}-\varepsilon_{1}-\varepsilon_{2}}\|u\|_{X^{0, \frac{1}{2}+\varepsilon_{1}, \delta}}^{3} .
$$

Note that the "loss" $\varepsilon_{1}$ comes from the fact that $b=\frac{1}{2}+\varepsilon_{1}$ is greater than $\frac{1}{2}$ and $\varepsilon_{2}$ comes from Lemma 3.2. Therefore, in proving local well-posedness via the fixed point argument, we require

$$
\delta^{\frac{1}{2}-\varepsilon_{1}-\varepsilon_{2}}\|u\|_{X^{0, \frac{1}{2}+, \delta}}^{2} \lesssim 1
$$


on the ball $\left\{u:\|u\|_{X^{0, \frac{1}{2}+, \delta}} \leq 2 C_{0}\left\|u_{0}\right\|_{L^{2}}\right\}$. Hence, we can choose $\delta \sim\left\|u_{0}\right\|_{L^{2}}^{-(4+\theta)}$ with $\theta=0+$.

Let $T>0$ and $\varepsilon>0$ be given, and we continue the argument from Subsection 1.4. First, in view of the large deviation estimate (1.15) $)$, choose $K \sim\left(\log \frac{1}{\varepsilon}\right)^{\frac{1}{2}}$ so that $\mathbb{P}\left(\left\|u_{0}(\omega)\right\|_{H^{s}} \geq\right.$ $K) \leq \frac{1}{2} \varepsilon$. In the following, we assume $\left\|u_{0}\right\|_{H^{s}} \leq K$. Now, fix $\delta \sim N^{(4+\theta) s} K^{-(4+\theta)}$ with $\theta=0+$. For fixed $\alpha \leq \frac{1}{2}, s=\alpha-\frac{1}{2}-<0$ is also fixed. Hence, we can write

$$
\delta \sim N^{4 s-} K^{-4-},
$$

Before proceeding further, we present an important proposition whose proof is given in the remaining part of the paper.

Proposition 5.2. Let $s=\alpha-\frac{1}{2}-$ with $\alpha \in\left(\frac{5}{12}, \frac{1}{2}\right]$. Given $T>0$ and $K>0$, there exists $N$ sufficiently large with $\delta \sim N^{4 s-} K^{-4-}$ such that the following holds. Suppose that

$$
\left\|u^{j}(t)\right\|_{X^{0, \frac{1}{2}+}{ }_{[(j-1) \delta, j \delta]}} \leq C N^{-s} K
$$

such that $\delta^{\frac{1}{2}-}\left\|u^{j}(t)\right\|_{X^{0, \frac{1}{2}+}{ }_{[(j-1) \delta, j \delta]}^{2}}^{2} \lesssim 1$ (see (15.2) ) for $j=1, \cdots,\left[\frac{T}{\delta}\right]$. Write the solution $v^{j}$ of the following difference equation:

$$
\left\{\begin{array}{l}
i \partial_{t} v^{j}-\partial_{x}^{2} v^{j} \pm\left(\mathcal{N}\left(u^{j}+v^{j}\right)-\mathcal{N}\left(u^{j}\right)\right)=0 \\
\left.v^{j}\right|_{t=(j-1) \delta}=\psi_{j-1}:=\sum_{|n|>N} \frac{g_{n}(\omega) e^{i(j-1) \delta n^{2}}}{\sqrt{1+|n|^{2 \alpha}}} e^{i n x}
\end{array}\right.
$$

as $v^{j}(t)=S(t-(j-1) \delta) \psi_{j-1}+w^{j}(t)$. Then, (5.5) is locally well-posed on the time interval $[(j-1) \delta, j \delta]$ except on a set of measure $e^{-\frac{1}{\delta^{c}}}$ for each $j=1, \cdots,\left[\frac{T}{\delta}\right]$. Moreover, we have the following bound on the nonlinear terms:

$$
\sum_{j=1}^{[T / \delta]}\left\|w^{j}(j \delta)\right\|_{L^{2}} \lesssim N^{-s} K
$$

Remark 5.3. In Proposition 5.2, we do not assume that $u^{j}$ is deterministic. In our application, $u^{j}$ is indeed random - not even independent from $\psi^{j-1}$ and $v^{j}$.

Now, we continue the proof of Proposition 5.1. Our choice of $\delta$ guarantees that (1.16) is well-posed on $[0, \delta]$ with the bound (1.17). Then, by Proposition [5.2, (1.18) is well-posed on $[0, \delta]$ except on a set of measure $e^{-\frac{1}{\delta^{c}}}$ with the bound (1.21), which in turn shows that (1.19) is well-posed on $[\delta, 2 \delta]$ with the bound (1.20).

Write the solution $v^{2}$ of (1.22) as $v^{2}(t)=S(t-\delta) \psi_{1}+w^{2}(t)$. It follows from (1.20) and Proposition 5.2 that (1.22) is well-posed on the time interval $[\delta, 2 \delta]$ except on a set of measure $e^{-\frac{1}{\delta c}}$. Moreover, we have

$$
\sum_{j=1}^{2}\left\|w^{j}(j \delta)\right\|_{L^{2}} \lesssim N^{-s} K
$$

At time $t=2 \delta$, write $u(2 \delta)=\phi_{2}+\psi_{2}$, where $\phi_{2}:=u^{2}(2 \delta)+w^{2}(2 \delta)$ and $\psi_{2}:=S(\delta) \psi_{1}=$ $S(2 \delta) \psi_{0}$. Then, (5.7) guarantees that the solution $u^{3}$ to

$$
\left\{\begin{array}{l}
i \partial_{t} u^{j}-\partial_{x}^{2} u^{j} \pm \mathcal{N}\left(u^{j}\right)=0 \\
\left.u^{j}\right|_{t=(j-1) \delta}=\phi_{j-1}
\end{array}\right.
$$


with $j=3$ satisfies

$$
\left\|u^{3}\right\|_{X^{0, \frac{1}{2}+}[2 \delta, 3 \delta]} \leq\left\|\phi_{0}\right\|_{L^{2}}+\sum_{j=1}^{2}\left\|w^{j}(j \delta)\right\|_{L^{2}} \lesssim N^{-s} K .
$$

Clearly, we can iterate this argument to show that (1.11) is well-posed on $[0, T]$, assuming (5.6) $)$ Lastly, note that the measure of the exceptional sets can be estimated by

$$
\left[\frac{T}{\delta}\right] e^{-\frac{1}{\delta^{c}}} \leq e^{\ln \frac{T}{\delta}-\frac{1}{\delta^{c}}} \leq e^{-\frac{1}{2} \frac{1}{\delta^{c}}}<\frac{1}{2} \varepsilon
$$

for sufficiently small $\delta>0$, i.e. for sufficiently large $N=N(T, \varepsilon)$. This completes the proof of Proposition 5.1.

5.2. Basic Setup. In the remaining part of the paper, we prove Proposition 5.2, In the following, fix $T>0$ and $K>0$, and let $s=\alpha-\frac{1}{2}-$ and (5.3):

$$
\delta \sim N^{4 s-} K^{-4-},
$$

where $N=N(T, K)$ to be determined later.

First, consider the following difference equation:

$$
\left\{\begin{array}{l}
i \partial_{t} v-\partial_{x}^{2} v \pm\left(\mathcal{N}\left(u^{0}+v\right)-\mathcal{N}\left(u^{0}\right)\right)=0 \\
\left.v\right|_{t=0}=\psi=\sum_{|n|>N} \frac{c_{n} g_{n}(\omega)}{\sqrt{1+|n|^{2 \alpha}}} e^{i n x}
\end{array}\right.
$$

where $\left|c_{n}\right|=1$ for all $n \in \mathbb{Z}$ and $u^{0}$ is a given function such that

$$
\left\|u^{0}(t)\right\|_{X^{0, \frac{1}{2}+, \delta}} \leq C N^{-s} K
$$

satisfying (5.2). Let $w$ denote the nonlinear part of the solution $v$ of (5.10). i.e. it is given by

$$
w(t):=w\left(t ; v, \psi, u^{0}\right)= \pm i \int_{0}^{t} S\left(t-t^{\prime}\right)\left(\mathcal{N}\left(u^{0}+v\right)-\mathcal{N}\left(u^{0}\right)\right)\left(t^{\prime}\right) d t^{\prime}
$$

for $t \in[0, \delta]$. From the linear estimate (4.1), we have

$$
\|w(\delta)\|_{L^{2}} \lesssim\left\|\eta_{\delta}(t) w\right\|_{X^{0, \frac{1}{2}+\varepsilon_{1}, \delta}} \lesssim\left\|\mathcal{N}\left(\widetilde{u^{0}}+\widetilde{v}\right)-\mathcal{N}\left(\widetilde{u^{0}}\right)\right\|_{X^{0,-\frac{1}{2}+\varepsilon_{1}}}
$$

for some small $\varepsilon_{1}>0$, where $\widetilde{u^{0}}$ and $\widetilde{v}$ are extensions of $u^{0}$ and $v$, respectively.

Suppose that we have

$$
\left\|\mathcal{N}\left(\widetilde{u^{0}}+\widetilde{v}\right)-\mathcal{N}\left(\widetilde{u^{0}}\right)\right\|_{X^{0,-\frac{1}{2}+}} \lesssim N^{3 s-\gamma}
$$

with some small $\gamma>0$ except on a set of measure $e^{-\frac{1}{\delta^{c}}}$ (for any extensions $\widetilde{u^{0}}$ and $\widetilde{v}$ of $u^{0}$ and $v$.) Then, it follows that the mapping $\Gamma$ defined by

$$
\Gamma v(t):=S(t) \psi+w\left(t ; v, \psi, u^{0}\right)
$$

is a contraction on $S(t) \psi^{\omega}+B$ on the time interval $[0, \delta]$ except on a set of measure $e^{-\frac{1}{\delta^{c}}}$, where $B$ denotes the ball of radius $\sim N^{3 s-\gamma}$ in $X_{[0, \delta]}^{0, \frac{1}{2}+}$. Moreover, from (5.3), (5.13), and (5.14), we have

$$
\frac{T}{\delta}\|w(\delta)\|_{L^{2}} \lesssim T \delta^{-1} N^{3 s-\gamma} \lesssim T K^{4+} N^{-s} N^{-\gamma+} \lesssim N^{-s} K
$$


for sufficiently large $N=N(T, K)$. Note that (5.13) and (5.14) imply only the boundedness of the map $\Gamma$ from $S(t) \psi^{\omega}+B$ into itself. In establishing the contraction property, one needs to consider the difference $\Gamma v_{1}-\Gamma v_{2}$ for $v_{1}, v_{2} \in S(t) \psi^{\omega}+B$. We omit details.

Finally, note that the bound (5.4) on $u^{j}$ is uniform in $j$ in Proposition [5.2. Hence, the above local well-posedness result can be applied to (5.5) on $[(j-1) \delta, j \delta]$ for $j=1, \cdots,\left[\frac{T}{\delta}\right]$, and moreover (5.6) follows from (5.16). Therefore, it remains to prove (5.14) for $\alpha \in\left(\frac{5}{12}, \frac{1}{2}\right]$ (and for large $N$.)

Note that (5.14) follows, once we prove

$$
\left\|\mathcal{N}_{j}\left(u_{1}, u_{2}, u_{3}\right)\right\|_{X^{0,-\frac{1}{2}+\varepsilon_{1}}} \lesssim N^{3 s-\gamma}, j=1,2,
$$

except on a set of measure $e^{-\frac{1}{\delta^{c}}}$, where $\mathcal{N}_{j}$ is as in (4.4) or (4.5), and $u_{j}$ is either of type

(I) linear part: random, less regular

$$
u_{j}(x, t)=S(t) \psi=\sum_{|n|>N} \frac{c_{n} g_{n}(\omega)}{\sqrt{1+|n|^{2 \alpha}}} e^{i\left(n x+n^{2} t\right)} \text { with }\left|c_{n}\right|=1, \text { or }
$$

(II) smoother:

(II.a) "high frequency" nonlinear part: small

$$
u_{j}=\widetilde{w} \text {, where } \widetilde{w} \text { is an extension of } w \text { with }\|w\|_{X^{0, \frac{1}{2}+\varepsilon_{1}, \delta}} \lesssim N^{3 s-\gamma},
$$

(II.b) "low frequency" input: large

$$
\begin{gathered}
u_{j}=\widetilde{u^{0}} \text {, where } \widetilde{u^{0}} \text { is an extension of } u^{0} \text { with }\left\|u^{0}\right\|_{X^{0, \frac{1}{2}+\varepsilon_{1}, \delta}} \lesssim N^{-s} K \\
\text { satisfying (15.2) }: \delta^{\frac{1}{2}-\varepsilon_{1}-\varepsilon_{2}}\left\|u^{0}\right\|_{X^{0, \frac{1}{2}+\varepsilon_{1}, \delta}}^{2} \lesssim 1,
\end{gathered}
$$

except for the case $u_{j}=u^{0}$ for all $j=1,2,3$. We may insert the smooth cutoff function $\eta_{\delta}$ supported on $[-2 \delta, 2 \delta]$ if necessary.

Note that $u^{0}$ has a much larger norm than $w$ since $s<0$. Thus, without loss of generality, we assume that $u_{j}=u^{0}$ if $u_{j}$ is of type (II), unless $u_{j}$ is of type (II) for all $j=1,2,3$. In the latter case, we may assume that two of $u_{j}$ 's are $u^{0}$ and the remaining $u_{j}$ is $w$., and it suffices to prove

$$
\left\|\mathcal{N}_{j}\left(u^{0}, u^{0}, w\right)\right\|_{X^{0,-\frac{1}{2}+\varepsilon_{1}}} \lesssim N^{3 s-\gamma}, j=1,2,
$$

assuming (5.2). In the following subsections, we prove (5.17) by separately estimating the contributions from $\mathcal{N}_{1}$ and $\mathcal{N}_{2}$. Indeed, except for Case (A) in Subsection 5.4 (namely with $u^{0}, u^{0}$, and $w$ ), we can prove (5.17) with $N^{3 s-\gamma-}$ instead of $N^{3 s-\gamma}$.

In the following, we write estimates directly with $\left\|u_{j}\right\|_{X^{s, b, \delta}}$ for simplicity of presentation, meaning that the same estimates hold with $\left\|\widetilde{u}_{j}\right\|_{X^{s, b}}$ for any extension $\widetilde{u}_{j}$ of $u_{j}$ (and thus we can take the infimum over $\widetilde{u}_{j}$.) See Remark 4.1 .

5.3. Estimate on $\mathcal{N}_{2}$. In this subsection, we prove the estimate (5.17) for $\mathcal{N}_{2}\left(u_{1}, u_{2}, u_{3}\right)$ defined in (4.5). We need to estimate

$$
\left\|\mathcal{N}_{2}\left(u_{1}, u_{2}, u_{3}\right)\right\|_{X^{0,-\frac{1}{2}+}}=\left\|\frac{1}{\left\langle\tau-n^{2}\right\rangle^{\frac{1}{2}-}} \int_{\tau=\tau_{1}-\tau_{2}+\tau_{3}} \widehat{u}_{1}\left(n, \tau_{1}\right) \overline{\widehat{u}_{2}\left(n, \tau_{2}\right)} \widehat{u}_{3}\left(n, \tau_{3}\right) d \tau_{1} d \tau_{2}\right\|_{l_{n}^{2} L_{\tau}^{2}}
$$


By Hölder inequality with $p$ large $\left(\frac{1}{2}=\frac{1}{2+}+\frac{1}{p}\right)$,

$$
\lesssim \sup _{n}\left\|\left\langle\tau-n^{2}\right\rangle^{-\frac{1}{2}+}\right\|_{L_{\tau}^{2+}}\left\|\int_{\tau=\tau_{1}-\tau_{2}+\tau_{3}} \widehat{u}_{1}\left(n, \tau_{1}\right) \overline{\widehat{u}_{2}\left(n, \tau_{2}\right)} \widehat{u}_{3}\left(n, \tau_{3}\right) d \tau_{1} d \tau_{2}\right\|_{l_{n}^{2} L_{\tau}^{p}} .
$$

In the following, we omit details if the computation is basically the same as in Subsection 4.3. Recall $\delta \sim N^{4 s-} K^{-4-}$ from (5.3). We assume that $N$ is sufficiently large in the following.

- Case (a): $u_{j}$ of type (II), $j=1, \ldots, 3$.

In this case, we prove (5.20). By Young and Hölder inequalities in $\tau$, followed by Hölder in $n, l_{n}^{2} \subset l_{n}^{6}$, and Lemma [3.2, we have

$$
\begin{aligned}
\text { (5.21) } & \lesssim \prod_{j=1}^{3}\left\|\left\langle\tau-n^{2}\right\rangle^{\frac{1}{6}+} \widehat{u}_{j}(n, \tau)\right\|_{l_{n}^{6} L_{\tau}^{2}} \leq \delta^{1-}\left\|u^{0}\right\|_{X^{0, \frac{1}{2}+, \delta}}^{2}\|w\|_{X^{0, \frac{1}{2}+, \delta}} \\
& \lesssim \delta^{\frac{1}{2}-} N^{3 s-\gamma} \lesssim N^{3 s-\gamma-}
\end{aligned}
$$

for $s \leq 0$.

- Case (b): $u_{j}$ of type (I), $j=1, \ldots, 3$.

By Lemma 3.4 and Young's inequality, we have

$$
\begin{aligned}
(5.21) & \lesssim \delta^{1-}\left\|\langle n\rangle^{-3 \alpha}\left|g_{n}(\omega)\right|^{3}\right\|_{l_{|n|>N}^{2}} \lesssim \delta^{1-\frac{3}{2} \beta-}\left\|\langle n\rangle^{-3 \alpha+3 \varepsilon}\right\|_{l_{|n|>N}^{2}} \\
& \lesssim \delta^{1-\frac{3}{2} \beta-} N^{-3 \alpha+\frac{1}{2}+3 \varepsilon} \lesssim N^{3 s-2 \alpha+} K^{-4-} \lesssim N^{3 s-\gamma-}
\end{aligned}
$$

for $\alpha>\frac{1}{2} \gamma>0$ outside an exceptional set of measure $<e^{-\frac{1}{\delta^{c}}}$.

- Case (c): Exactly two $u_{j}$ 's of type (I). Say $u_{1}(\mathrm{I}), u_{2}(\mathrm{I})$, and $u_{3}(\mathrm{II})$.

By Young's inequality and Lemmata 3.2 and 3.4, we have

$$
\begin{aligned}
\text { (5.21) } & \lesssim \delta^{\frac{1}{2}-}\left(\sup _{|n|>N}\langle n\rangle^{-2 \alpha}\left|g_{n}\right|^{2}\right)\left\|\widehat{u^{0}}(n, \tau)\right\|_{l_{n}^{2} L_{\tau}^{2}} \lesssim \delta^{1-\beta-} N^{-2 \alpha+2 \varepsilon}\left\|u^{0}\right\|_{X^{0, \frac{1}{2}+, \delta}} \\
& \lesssim N^{3 s-2 \alpha+} K^{-3-} \lesssim N^{3 s-\gamma-}
\end{aligned}
$$

for $\alpha>\frac{1}{2} \gamma>0$ outside an exceptional set of measure $<e^{-\frac{1}{\delta^{c}}}$.

- Case (d): Exactly one $u_{j}$ of type (I). Say $u_{1}(\mathrm{I}), u_{2}(\mathrm{II})$, and $u_{3}(\mathrm{II})$.

By Young's inequality, followed by Hölder inequality in $n\left(\frac{1}{2}=\frac{1}{4}+\frac{1}{4}\right)$ and in $\tau\left(\frac{3}{4}=\frac{1}{2}+\frac{1}{4}\right)$ and Lemmata 3.2 and 3.4 , we have

$$
\begin{aligned}
\text { (15.21) } & \lesssim \delta^{\frac{1}{2}-}\left(\sup _{|n|>N}\langle n\rangle^{-\alpha}\left|g_{n}\right|\right)\|\| \widehat{u^{0}}(n, \tau)\left\|_{L_{\tau}^{\frac{4}{3}}}^{2}\right\|_{l_{n}^{2}} \\
& \lesssim \delta^{\frac{1}{2}-\frac{\beta}{2}-} N^{-\alpha+\varepsilon} \sup _{n}\left\|\left\langle\tau-n^{2}\right\rangle^{-\frac{1}{4}-}\right\|_{L_{\tau}^{4}}^{2}\left\|\left\langle\tau-n^{2}\right\rangle^{\frac{1}{4}+} \widehat{u^{0}}(n, \tau)\right\|_{l_{n}^{4} L_{\tau}^{2}}^{2} \\
& \lesssim \delta^{1-\frac{\beta}{2}-} N^{-\alpha+\varepsilon}\left\|\left\langle\tau-n^{2}\right\rangle^{\frac{1}{2}} \widehat{v}_{j}(n, \tau)\right\|_{l_{n}^{4} L_{\tau}^{2}}^{2} \lesssim N^{2 s-\alpha+} K^{-2-} \lesssim N^{3 s-\gamma-}
\end{aligned}
$$

for $\alpha>\frac{1}{4}+\frac{1}{2} \gamma>\frac{1}{4}$ outside an exceptional set of measure $<e^{-\frac{1}{\delta^{c}}}$.

5.4. Estimate on $\mathcal{N}_{1}$ : High Modulation Cases. In the next two subsections, we prove the main part of the estimate (5.17):

$$
\left\|\mathcal{N}_{1}\left(u_{1}, u_{2}, u_{3}\right)\right\|_{X^{0,-\frac{1}{2}+\varepsilon_{1}, \delta}} \lesssim N^{3 s-\gamma}
$$

for some small $\gamma>0$, where $\mathcal{N}_{1}$ is as in (4.4) and $u_{j}$ is of type (I) or (II). Once again, we omit details in the following when the computation basically follows from Subsection 4.4. 
Using duality, we can estimate (5.22) by

$$
\iint u^{1} u^{2} u^{3} \cdot v d x d t
$$

where $\|v\|_{X^{0, \frac{1}{2}-\varepsilon_{1}, \delta}} \leq 1$ (with the complex conjugate on an appropriate $u^{j}$.) We assume that $N$ is sufficiently large in the following.

- Case (A): $u^{1}$ and $u^{2}$ are of type (II).

Suppose that $u^{3}$ is of type (II). In this case, we prove (5.20) instead of (5.22). By Lemmata 3.3 (a) and 3.2 with (5.18) and (5.19) (also see (5.2)), we have

$$
\begin{aligned}
(5.23) & \lesssim \delta^{\frac{1}{2}-\varepsilon_{1}-\varepsilon_{2}}\left\|u^{0}\right\|_{X^{0, \frac{1}{2}, \delta}}^{2}\|w\|_{X^{0, \frac{1}{2}, \delta}}\|v\|_{X^{0, \frac{1}{2}-\varepsilon_{1}, \delta}} \\
& \lesssim\|w\|_{X^{0, \frac{1}{2}, \delta}} \lesssim N^{3 s-\gamma} .
\end{aligned}
$$

Next, suppose that $u^{3}$ is of type (I) i.e. $u^{3}=S(t) u_{0}$. In this case, we do not need to apply dyadic decomposition on $u^{1}$ and $u^{2}$. Namely, for a fixed dyadic block $N^{3}$ for $u^{3}$ of type (I), with a slight abuse of notation, we use $u^{1}$ and $u^{2}$ to denote the sums of $u^{j}$ over the dyadic blocks $N^{j} \geq N^{3}, j=1,2$.

By Lemma 3.3 (b) with $p$ large followed by Lemma 3.5, we have

$$
(5.23) \lesssim\left(N^{3}\right)^{\frac{1}{2}-\alpha+}\left\|u^{0}\right\|_{X^{0, \frac{1}{4}+, \delta}}^{2}\|v\|_{X^{0, \frac{1}{4}+, \delta}}
$$

outside an exceptional set of measure $<e^{-\frac{1}{\delta^{c}}}$. If $\left\langle\tau_{j}-n_{j}^{2}\right\rangle^{\frac{1}{4}-} \gtrsim\left(N^{3}\right)^{\frac{1}{2}-\alpha+} N^{-3 s+\gamma+\widetilde{\varepsilon}}$ for $u_{j}$ of type (II), or if $\left\langle\tau-n^{2}\right\rangle^{\frac{1}{4}-} \gtrsim\left(N^{3}\right)^{\frac{1}{2}-\alpha+} N^{-3 s+\gamma+\widetilde{\varepsilon}}$, then it follows from Lemma 3.2, (5.3), and (5.19) that

$$
(5.23) \lesssim \delta^{\frac{1}{2}-} N^{-2 s} K^{2} N^{3 s-\gamma-\widetilde{\varepsilon}} \lesssim N^{3 s-\gamma-}
$$

for $N$ sufficiently large. Recall $N^{3}>N, s=\alpha-\frac{1}{2}-$, and $\gamma=0+$.

Hence, it remains to estimate the contribution to (5.22) from the region satisfying

$$
\left\langle\tau-n^{2}\right\rangle \ll\left(N^{3}\right)^{8-16 \alpha+} \text {, and }\left\langle\tau_{j}-n_{j}^{2}\right\rangle \ll\left(N^{3}\right)^{8-16 \alpha+} \text { if } u_{j} \text { of type (II) }
$$

in the following.

- Case (B): $u^{1}$ of type (II), and $u^{2}$ of type (I).

In this case, we do not need to apply dyadic decomposition on $u^{1}$. Namely, for a fixed dyadic block $N^{2}$ for $u^{2}$ of type (I), we use $u^{1}$ to denote the sum of $u^{1}$ over the dyadic blocks $N^{1} \geq N^{2}$.

○ Subcase (B.1): $u^{3}$ is of type (II). By Lemma 3.3 (b) with $p$ large followed by Lemma 3.5, we have

$$
(\underline{5.23)}) \lesssim\left(N^{2}\right)^{\frac{1}{2}-\alpha+}\left\|u^{0}\right\|_{X^{0, \frac{1}{4}+, \delta}}^{2}\|v\|_{X^{0, \frac{1}{4}+, \delta}}
$$

outside an exceptional set of size $<e^{-\frac{1}{\delta^{c}}}$. If $\left\langle\tau_{j}-n_{j}^{2}\right\rangle^{\frac{1}{4}-} \gtrsim\left(N^{2}\right)^{\frac{1}{2}-\alpha+} N^{-3 s+\gamma+}$ for $u_{j}$ of type (II), or if $\left\langle\tau-n^{2}\right\rangle^{\frac{1}{4}-} \gtrsim\left(N^{2}\right)^{\frac{1}{2}-\alpha+} N^{-3 s+\gamma+}$, then (5.22) follows as in Case (A).

Hence, it remains to estimate the contribution to (5.22) from the region satisfying

$$
\left\langle\tau-n^{2}\right\rangle \ll\left(N^{2}\right)^{8-16 \alpha+} \text {, and }\left\langle\tau_{j}-n_{j}^{2}\right\rangle \ll\left(N^{2}\right)^{8-16 \alpha+} \text { if } u_{j} \text { of type (II). }
$$

○ Subcase (B.2): $u^{3}$ is of type (I). By Lemma 3.3 (b) with $p$ large followed by Lemma 3.5 , we have

$$
\left(\underline{5.23)} \lesssim\left(N^{2}\right)^{1-2 \alpha+}\left\|u^{0}\right\|_{X^{0,0+, \delta}}\|v\|_{X^{0,0+, \delta}}\right.
$$


outside an exceptional set of measure $<e^{-\frac{1}{\delta^{c}}}$. If $\left(\sigma^{1}\right)^{\frac{1}{2}-} \gtrsim\left(N^{2}\right)^{1-2 \alpha+} N^{-2 s+\gamma+}$ or if $\langle\tau-$ $\left.n^{2}\right\rangle^{\frac{1}{2}-} \gtrsim\left(N^{2}\right)^{1-2 \alpha+} N^{-2 s+\gamma+}$, then (5.22) follows from Lemma 3.2. (5.3), and (5.19).

Hence, it remains to estimate the contribution to (5.22) from the region satisfying

$$
\left\langle\tau-n^{2}\right\rangle \ll\left(N^{2}\right)^{4-8 \alpha+} \text {, and }\left\langle\tau_{j}-n_{j}^{2}\right\rangle \ll\left(N^{2}\right)^{4-8 \alpha+} \text { if } u_{j} \text { of type (II). }
$$

- Case (C): $u^{1}$ of type (I), and $u^{2}, u^{3}$ of type (II).

Dyadically decompose all the spatial frequencies. Suppose $\left\langle\tau-n^{2}\right\rangle \gg \max \left(\sigma^{2}, \sigma^{3}\right)$. By Lemma 3.3 (c) with $p$ large, Lemmata 3.5 and 3.2, and (5.19), we have

$$
\text { (5.23) } \lesssim\left(N^{1}\right)^{\frac{1}{2}-\alpha+}\left\|u^{0}\right\|_{X^{0, \frac{3}{8}, \delta}}^{2}\|v\|_{X^{0,0+, \delta}} \lesssim \delta^{\frac{1}{4}-}\left(N^{1}\right)^{\frac{1}{2}-\alpha+} N^{-2 s} K^{2}\|v\|_{X^{0,0+}}
$$

outside an exceptional set of measure $<e^{-\frac{1}{\delta^{c}}}$. Hence, as before, (5.22) follows as long as $\left\langle\tau-n^{2}\right\rangle^{\frac{1}{2}-} \gtrsim\left(N^{1}\right)^{\frac{1}{2}-\alpha+} N^{-4 s+\gamma+}$. Similar results hold if $\sigma^{2} \gg \max \left(\sigma^{3},\left\langle\tau-n^{2}\right\rangle\right)$ or $\sigma^{3} \gtrsim \max \left(\sigma^{2},\left\langle\tau-n^{2}\right\rangle\right)$.

Hence, it remains to estimate the contribution to (5.22) from the region satisfying

$$
\left\langle\tau-n^{2}\right\rangle \ll\left(N^{1}\right)^{5-10 \alpha+} \text {, and }\left\langle\tau_{j}-n_{j}^{2}\right\rangle \ll\left(N^{1}\right)^{5-10 \alpha+} \text { if } u_{j} \text { of type (II). }
$$

- Case (D): $u^{1}$ of type (I), and either $u^{2}(\mathrm{I}), u^{3}(\mathrm{II})$ or $u^{2}(\mathrm{II}), u^{3}(\mathrm{I})$.

Suppose that $u^{2}$ is of type (I) and that $u^{3}$ is of type (II). Moreover, suppose $\left\langle\tau-n^{2}\right\rangle \gg \sigma^{3}$. By Lemma 3.3 (c) with $p$ large, Lemmata 3.5 and 3.2, and (5.19), we have

$$
(\underline{5.23)}) \lesssim\left(N^{1}\right)^{1-2 \alpha+}\left\|u^{0}\right\|_{X^{0,0+}}\|v\|_{X^{0,0}} \lesssim \delta^{\frac{1}{2}-}\left(N^{1}\right)^{1-2 \alpha+} N^{-s} K\|v\|_{X^{0,0}}
$$

outside an exceptional set of measure $<e^{-\frac{1}{\delta^{c}}}$. Hence, (5.22) follows as long as $\left\langle\tau-n^{2}\right\rangle^{\frac{1}{2}-} \gtrsim$ $\left(N^{1}\right)^{1-2 \alpha+} N^{-2 s+\gamma+}$. Similar results hold if $\sigma^{3} \gtrsim\left\langle\tau-n^{2}\right\rangle$, (or $u^{2}$ is of type (II) and $u^{3}$ is of type (I).)

Hence, it remains to estimate the contribution to (5.22) from the region satisfying

$$
\left\langle\tau-n^{2}\right\rangle \ll\left(N^{1}\right)^{4-8 \alpha+} \text {, and }\left\langle\tau_{j}-n_{j}^{2}\right\rangle \ll\left(N^{1}\right)^{4-8 \alpha+} \text { if } u_{j} \text { of type (II). }
$$

Summary: By repeating the computation in Subsection 4.4, we can reduce the estimate into the following two cases (with $\theta=0+$ ):.

- $u^{1}$ is of type (II): By (4.14) and (4.15), we can bound (5.22) as follows:

$$
\left(\underline{5.22)} \lesssim \delta^{\theta} M\left(N, N^{2}, N^{3}\right)\left(\sum_{n}\left|\sum_{\substack{n=n_{1}-n_{2}+n_{3} \\ n_{2} \neq n_{1}, n_{3} \\ n^{2}=n_{1}^{2}-n_{2}^{2}+n_{3}^{2}+\mu}} a_{1}\left(n_{1}\right) \overline{a_{2}\left(n_{2}\right)} a_{3}\left(n_{3}\right)\right|^{2}\right)^{\frac{1}{2}}\right. \text {, }
$$

where $\sum_{n}\left|a^{1}(n)\right|^{2} \leq 1, a^{2}(n)=\frac{g_{n}(\omega)}{1+|n|^{\alpha}}, a^{3}(n)=\frac{g_{n}(\omega)}{1+|n|^{\alpha}}$ or $\sum_{|n| \sim N^{3}}\left|a^{3}(n)\right|^{2} \leq 1$, and

Case (A): $\quad M\left(N, N^{2}, N^{3}\right)=\left(N^{3}\right)^{0+} N^{-2 s}$ and $|\mu| \ll\left(N^{3}\right)^{8-16 \alpha+}$

Subcase (B.1): $\quad M\left(N, N^{2}, N^{3}\right)=\left(N^{2}\right)^{0+} N^{-2 s}$ and $|\mu| \ll\left(N^{2}\right)^{8-16 \alpha+}$

Subcase (B.2): $\quad M\left(N, N^{2}, N^{3}\right)=\left(N^{2}\right)^{0+} N^{-s}$ and $|\mu| \ll\left(N^{2}\right)^{4-8 \alpha+}$.

Note that we did not apply dyadic decomposition on $N^{1}$. 
- $u^{1}$ is of type (I): By (4.14) and (4.15), we can bound (5.22) as follows:

$$
(\underline{5.22}) \lesssim \delta^{\theta}\left(N^{1}\right)^{0+} M(N)\left(\sum_{|n| \lesssim N^{1}}\left|\sum_{\substack{n=n_{1}-n_{2}+n_{3} \\ n_{2} \neq n_{1}, n_{3} \\ n^{2}=n_{1}^{2}-n_{2}^{2}+n_{3}^{2}+\mu}} a_{1}\left(n_{1}\right) \overline{a_{2}\left(n_{2}\right)} a_{3}\left(n_{3}\right)\right|^{2}\right)^{\frac{1}{2}},
$$

where $a^{1}(n)=\frac{g_{n}(\omega)}{1+|n|^{\alpha}}, a^{j}(n)=\frac{g_{n}(\omega)}{1+|n|^{\alpha}}$ or $\sum_{|n| \sim N^{j}}\left|a^{j}(n)\right|^{2} \leq 1$ for $j=2,3$, and

Case (C): $\quad M(N)=N^{-2 s}$ and $|\mu| \ll\left(N^{1}\right)^{5-10 \alpha+}$

Case (D): $\quad M(N)=N^{-s}$ and $|\mu| \ll\left(N^{1}\right)^{4-8 \alpha+}$

All type (I): $\quad M(N)=1 \quad$ and $|\mu| \lesssim\left(N^{1}\right)^{2}$.

Note that all the spatial frequencies are dyadically decomposed.

By symmetry between $u_{1}$ and $u_{3}$, we assume $\left|n_{1}\right| \sim N^{1}$ or $\left|n_{2}\right| \sim N^{1}$ in the following. Moreover, in Subcase (B.2) and Case (D), we may assume that $\left|n_{1}\right| \sim N^{1}$. If not, say, we have $\left|n_{2}\right|>10\left(\left|n_{1}\right|+\left|n_{3}\right|\right)$. Then, $|\mu| \sim\left|\left(n_{2}-n_{1}\right)\left(n_{2}-n_{3}\right)\right| \sim\left|n_{2}\right|^{2} \sim\left(N^{1}\right)^{2}$ by (3.1). In these two cases, we have $|\mu| \ll\left(N^{j}\right)^{4-8 \alpha+} \ll\left(N^{1}\right)^{2}$ as long as $\alpha>\frac{1}{4}$. i.e. we would have a contradiction.

Lastly, we list all the different cases as before. We consider these cases in details in the next subsection.

- $n_{1}=N^{1}$ :

Case (a): $n_{1}=N^{1}\left(\right.$ II), $n_{2}=N^{2}(\mathrm{I}), n_{3}=N^{3}\left(\right.$ II) or $n_{2}=N^{3}(\mathrm{I}), n_{3}=N^{2}($ II)

Case (b): $n_{1}=N^{1}(\Pi), n_{2}=N^{3}(\Pi), n_{3}=N^{2}(\mathrm{I})$ or $n_{2}=N^{2}(\Pi), n_{3}=N^{3}(\mathrm{I})$

Case (c): $n_{1}=N^{1}(\mathrm{I}), n_{2}=N^{2}\left(\right.$ II),$n_{3}=N^{3}($ II)

Case (d): $n_{1}=N^{1}(\mathrm{I}), n_{2}=N^{3}(\Pi), n_{3}=N^{2}($ II)

Case (e): $n_{1}=N^{1}\left(\right.$ II) $, n_{2}=N^{2}(\mathrm{I}), n_{3}=N^{3}(\mathrm{I})$

Case (f): $n_{1}=N^{1}(\Pi), n_{2}=N^{3}(\mathrm{I}), n_{3}=N^{2}(\mathrm{I})$

Case (g): $n_{1}=N^{1}(\mathrm{I}), n_{2}=N^{2}(\mathrm{I}), n_{3}=N^{3}(\mathrm{I})$

Case (h): $n_{1}=N^{1}(\mathrm{I}), n_{2}=N^{3}(\mathrm{I}), n_{3}=N^{2}(\mathrm{II})$

Case (i): $n_{1}=N^{1}(\mathrm{I}), n_{2}=N^{2}(\mathrm{I}), n_{3}=N^{3}(\mathrm{I})$

Case (j): $n_{1}=N^{1}(\mathrm{I}), n_{2}=N^{3}(\mathrm{I}), n_{3}=N^{2}(\mathrm{I})$

Case (k): All type (I)

- $n_{2}=N^{1}$ :

Case (a'): $n_{2}=N^{1}\left(\right.$ II) $, n_{1}=N^{2}(\mathrm{I}), n_{3}=N^{3}(\Pi)$ or $n_{1}=N^{3}(\mathrm{I}), n_{3}=N^{2}(\Pi)$

Case (b'): $n_{2}=N^{1}\left(\right.$ II), $n_{1}=N^{3}\left(\right.$ II), $n_{3}=N^{2}(\mathrm{I})$ or $n_{1}=N^{2}\left(\right.$ II),$n_{3}=N^{3}(\mathrm{I})$

Case (c'): $n_{2}=N^{1}(\mathrm{I}), n_{1}=N^{2}\left(\right.$ II),$n_{3}=N^{3}($ II)

Case (d'): $n_{2}=N^{1}(\mathrm{I}), n_{1}=N^{3}(\Pi), n_{3}=N^{2}(\Pi)$

Case $\left(\mathrm{k}^{\prime}\right)$ : All type $(\mathrm{I})$

5.5. Estimate on $\mathcal{N}_{1}$ : Low Modulation Cases. As before, we use $|n|^{\alpha}$ for $1+|n|^{\alpha}$ and drop a complex conjugate on $u_{2}$ when it plays no significant role. Let $A_{n}$ and $B_{n}$ be as in Subsection 4.4, Recall

$$
\mu=2\left(n_{2}-n_{1}\right)\left(n_{2}-n_{3}\right)=2\left(n-n_{1}\right)\left(n-n_{3}\right)
$$

from (4.21), $s=\alpha-\frac{1}{2}-$, and $N_{j}>N$ if $u_{j}$ is of type (I). 
- Cases (k), (k'): $u_{1}, u_{2}, u_{3}$ of type (I). In this case, we have

$$
(\underline{5.30}) \lesssim \delta^{\theta}\left(N^{1}\right)^{0+}\left(\sum_{|n| \lesssim N^{1}}\left|\sum_{B_{n}} \frac{g_{n_{1}}}{\left|n_{1}\right|^{\alpha}} \frac{\overline{g_{n_{2}}}}{\left|n_{2}\right|^{\alpha}} \frac{g_{n_{3}}}{\left|n_{3}\right|^{\alpha}}\right|^{2}\right)^{\frac{1}{2}} .
$$

Note that we have $N_{1}, N_{2}, N_{3}>N$. First, we consider the contribution from $n_{1} \neq n_{3}$. As in Subsection 4.4, by (4.23) and Lemma 3.1, we have

$$
\begin{aligned}
\operatorname{RHS} \text { of (5.31) } & \lesssim \delta^{\theta-\frac{3}{2} \beta}\left(N^{1}\right)^{\frac{3}{2} \varepsilon+}\left(\sum_{|n| \lesssim N^{1}} \sum_{C_{n}} \frac{1}{\left|n_{1}\right|^{2 \alpha}\left|n_{2}\right|^{2 \alpha}\left|n_{3}\right|^{2 \alpha}}\right)^{\frac{1}{2}} \\
& \lesssim \delta^{\theta-\frac{3}{2} \beta}\left(N^{1}\right)^{-\alpha+\frac{3}{2} \varepsilon+}\left(N^{2}\right)^{-\alpha}\left(N^{3}\right)^{-\alpha+\frac{1}{2}} \lesssim N^{-3 \alpha+\frac{1}{2}+} \leq N^{3 s-\gamma-} \prod_{j=1}^{3} N_{j}^{0-}
\end{aligned}
$$

for $\alpha>\frac{1}{3}+\frac{1}{6} \gamma>\frac{1}{3}$ and sufficiently large $N$ outside an exceptional set of measure < $\left(N^{1}\right)^{0-} e^{-\frac{1}{\delta^{c}}}$ as in (4.24).

The contribution from $n_{1}=n_{3}$ follows as in (4.25). By Lemmata 3.1 and 3.4, we have

$$
\text { RHS of (5.31) } \lesssim \delta^{\theta-\frac{3}{2} \beta}\left(N^{1}\right)^{0+} N_{1}^{-2 \alpha+2 \varepsilon} N_{2}^{-\alpha+\varepsilon}\left(N^{3}\right)^{\frac{1}{2}} \leq N^{3 s-\gamma-} \prod_{j=1}^{3} N_{j}^{0-}
$$

for $\alpha>\frac{1}{3}+\frac{1}{6} \gamma>\frac{1}{3}$ and sufficiently large $N$ outside an exceptional set of measure $<e^{-\frac{1}{\delta^{c}}}$.

- Case (a) : (Cases (b), (a'), and (b') can be treated in a similar way by replacing $n_{2}$ with $n_{3}, n_{2}$ with $n_{1}$, and $\left(n_{1}, n_{2}, n_{3}\right)$ with $\left(n_{2}, n_{3}, n_{1}\right)$, respectively.)

In this case, we have $\mu=2\left(n_{2}-n_{1}\right)\left(n_{2}-n_{3}\right)=o\left(\left(N_{2}\right)^{8-16 \alpha+}\right)$. Thus, by Lemma 3.4, Cauchy-Schwarz inequality, and (4.26) as before, we have

$$
\begin{aligned}
(\text { (5.29) } & \lesssim \delta^{\theta-\frac{\beta}{2}}\left(N_{2}\right)^{-\alpha+\frac{1}{2} \varepsilon+} N^{-2 s}\left(\sum_{n}\left(\sum_{A_{n}}\left|a_{1}\left(n_{1}\right)\right|^{2}\left|a_{3}\left(n_{3}\right)\right|^{2}\right)\left(\sum_{A_{n}} 1\right)\right)^{\frac{1}{2}} \\
& \lesssim \delta^{\theta-\frac{\beta}{2}} N_{2}^{-\alpha+\varepsilon+} N^{-2 s}\left(\sum_{n} \sum_{A_{n}}\left|a_{1}\left(n_{1}\right)\right|^{2}\left|a_{3}\left(n_{3}\right)\right|^{2}\right)^{\frac{1}{2}} \\
& \lesssim \delta^{\theta-\frac{\beta}{2}} N_{2}^{-\alpha+\varepsilon+} N^{-2 s} \leq N^{3 s-\gamma-} N_{2}^{0-} N_{3}^{0-}
\end{aligned}
$$

for $\alpha>\frac{5}{12}+\frac{1}{6} \gamma>\frac{5}{12}$ and sufficiently large $N$ outside an exceptional set of measure $<e^{-\frac{1}{\delta^{c}}}$.

- Case (c): (Case (d) can be treated in a similar way by replacing $n_{2}$ and $n_{3}$.)

By Lemma 3.4 and Hölder inequality on $n_{3}$ in the inner sum,

$$
\text { (15.30) } \lesssim \delta^{\theta-\frac{\beta}{2}} N_{1}^{-\alpha+\varepsilon+} N^{-2 s}\left(\sum_{|n| \lesssim N^{1}} \sum_{B_{n}}\left|a_{2}\left(n_{2}\right)\right|^{2}\right)^{\frac{1}{2}}
$$

outside an exceptional set of measure $<e^{-\frac{1}{\delta^{c}}}$. For fixed $n_{2}$, it follows from (the proof of) Lemma 3.1 that there are at most $N_{1}^{0+}$ terms in the sum. Hence, we have

$$
\left(\text { (5.30) } \lesssim \delta^{\theta-\frac{\beta}{2}} N_{1}^{-\alpha+\varepsilon+} N^{-2 s} \leq N^{3 s-\gamma-} \prod_{j=1}^{3} N_{j}^{0-}\right.
$$

for $\alpha>\frac{5}{12}+\frac{1}{6} \gamma>\frac{5}{12}$ and sufficiently large $N$. 
- Case (e) : (Case (f) is basically the same.)

In this case, we have $|\mu|=\left|2\left(n_{2}-n_{1}\right)\left(n_{2}-n_{3}\right)\right| \ll N_{2}^{4-8 \alpha+}$. This implies that $|n|,\left|n_{1}\right|,\left|n_{3}\right| \lesssim N_{2}^{q}$ for some $q>0$ since $n_{2} \neq n_{1}, n_{3}$. Then, by Lemma 3.4, Cauchy-Schwarz inequality, and (4.26) as before, we have

$$
\begin{aligned}
([5.29) & \lesssim \delta^{\theta-\beta} N_{2}^{-\alpha+\varepsilon+} N_{3}^{-\alpha} N^{-s}\left(\sum_{|n| \lesssim N_{2}^{q}}\left(\sum_{A_{n}}\left|a_{1}\left(n_{1}\right)\right|^{2}\right)\left(\sum_{A_{n}} 1\right)\right)^{\frac{1}{2}} \\
& \lesssim \delta^{\theta-\beta} N_{2}^{-\alpha+\frac{3}{2} \varepsilon+} N_{3}^{-\alpha} N^{-s}\left(\sum_{|n| \lesssim N_{2}^{q}} \sum_{A_{n}}\left|a_{1}\left(n_{1}\right)\right|^{2}\right)^{\frac{1}{2}} \\
& \lesssim \delta^{\theta-\beta} N_{2}^{-\alpha+2 \varepsilon+} N_{3}^{-\alpha} N^{-s} \leq N^{3 s-\gamma-} N_{2}^{0-} N_{3}^{0-}
\end{aligned}
$$

for $\alpha>\frac{1}{3}+\frac{1}{6} \gamma>\frac{1}{3}$ and sufficiently large $N$ outside an exceptional set of measure $<e^{-\frac{1}{\delta^{c}}}$.

- Case (g) : (Cases (h), (i), (j) are basically the same.)

By Lemma 3.4 and Cauchy-Schwarz inequality as before, we have

$$
\begin{aligned}
([5.30) & \lesssim \delta^{\theta-\beta} N_{1}^{-\alpha+\varepsilon+} N_{2}^{-\alpha+\frac{1}{2} \varepsilon} N^{-s}\left(\sum_{|n| \lesssim N_{1}} \sum_{A_{n}}\left|a_{3}\left(n_{3}\right)\right|^{2}\right)^{\frac{1}{2}} \\
& \lesssim \delta^{\theta-\beta} N_{1}^{-\alpha+\frac{3}{2} \varepsilon+} N_{2}^{-\alpha+\frac{1}{2} \varepsilon} N^{-s} \leq N^{3 s-\gamma-} \prod_{j=1}^{3} N_{j}^{0-}
\end{aligned}
$$

for $\alpha>\frac{1}{3}+\frac{1}{6} \gamma>\frac{1}{3}$ and sufficiently large $N$ outside an exceptional set of measure $<e^{-\frac{1}{\delta^{c}}}$.

Remark 5.4. It is worthwhile to note that the worst case occurs:

- for all type (I) in the local theory.

- for one (I) and two (II.b) in the global theory.

These cases yield the conditions on the values of $\alpha$ in Theorems 1 and 2 ,

Acknowledgments: The authors would like to thank Prof. Kenji Nakanishi for the conversation at Institut Henri Poincaré. They are also grateful to Prof. Nicolas Burq for remarks on a preliminary draft. Lastly, they would like to express their gratitude to the anonymous referees for thoughtful comments that have improved this paper.

\section{REFERENCES}

[1] Bényi, Á.; Oh, T. Modulation spaces, Wiener amalgam spaces, and Brownian motions, to appear in Adv. Math.

[2] Bourgain, J. Fourier transform restriction phenomena for certain lattice subsets and applications to nonlinear evolution equations. I. Schrödinger equations, Geom. Funct. Anal. 3 (1993), no. 2, 107-156.

[3] Bourgain, J. Periodic nonlinear Schrödinger equation and invariant measures, Comm. Math. Phys. 166 (1994), no. 1, 1-26.

[4] Bourgain, J. On the Cauchy and invariant measure problem for the periodic Zakharov system, Duke Math. J. 76 (1994), no. 1, 175-202.

[5] Bourgain, J. Invariant measures for the 2 D-defocusing nonlinear Schrödinger equation, Comm. Math. Phys. 176 (1996), no. 2, 421-445.

[6] Bourgain, J. Refinements of Strichartz' inequality and applications to 2D-NLS with critical nonlinearity, Internat. Math. Res. Notices 1998, no. 5, 253-283.

[7] Bourgain, J. Nonlinear Schrödinger equations, Hyperbolic equations and frequency interactions (Park City, UT, 1995), 3-157, IAS/Park City Math. Ser., 5, Amer. Math. Soc., Providence, RI, 1999. 
[8] Burq, N.; Gérard, P.; Tzvetkov, N. An instability property of the nonlinear Schrödinger equation on $S^{d}$, Math. Res. Lett. 9 (2002), no. 2-3, 323-335.

[9] Burq, N.; Tzvetkov, N. Invariant measure for a three dimensional nonlinear wave equation, Int. Math. Res. Not. IMRN 2007, no. 22, Art. ID rnm108, 26 pp.

[10] Burq, N.; Tzvetkov, N. Random data Cauchy theory for supercritical wave equations. I. Local theory, Invent. Math. 173 (2008), no. 3, 449-475.

[11] Burq, N.; Tzvetkov, N. Random data Cauchy theory for supercritical wave equations. II. A global existence result, Invent. Math. 173 (2008), no. 3, 477-496.

[12] Christ, M. Power series solution of a nonlinear Schrödinger equation, Mathematical aspects of nonlinear dispersive equations, 131-155, Ann. of Math. Stud., 163, Princeton Univ. Press, Princeton, NJ, 2007.

[13] Christ, M.; Colliander, J.; Tao, T. Instability of the periodic nonlinear Schrödinger equation, arXiv:math/0311227 v1 [math.AP].

[14] Christ, M.; Colliander, J.; Tao, T. Asymptotics, frequency modulation, and low regularity ill-posedness for canonical defocusing equations, Amer. J. Math. 125 (2003), no. 6, 1235-1293.

[15] Ginibre, J.; Tsutsumi, Y.; Velo, G. On the Cauchy problem for the Zakharov system, J. Funct. Anal. 151 (1997), no. 2, 384-436.

[16] Grünrock, A.; Herr, S. Low regularity local well-posedness of the derivative nonlinear Schrödinger equation with periodic initial data, SIAM J. Math. Anal. 39 (2008), no. 6, 1890-1920.

[17] Hardy, G. H.; Wright, E. M. An introduction to the theory of numbers, Fifth edition. The Clarendon Press, Oxford University Press, New York, 1979. xvi+426 pp.

[18] Janson, S. Gaussian Hilbert Spaces, Cambridge Tracts in Mathematics, 129. Cambridge University Press, Cambridge, 1997. $\mathrm{x}+340$ pp.

[19] Kenig, C.; Ponce, G.; Vega, L. On the ill-posedness of some canonical dispersive equations, Duke Math. J. 106 (2001), no. 3, 617-633.

[20] Klainerman, S.; Selberg, S. Bilinear estimates and applications to nonlinear wave equations, Commun. Contemp. Math. 4 (2002), no. 2, 223-295.

[21] Koch, H.; Tataru, D. A priori bounds for the $1 D$ cubic NLS in negative Sobolev spaces, Int. Math. Res. Not. IMRN 2007, no. 16, Art. ID rnm053, 36 pp.

[22] Kuo, H. Gaussian measures in Banach spaces, Lecture Notes in Mathematics, Vol. 463. Springer-Verlag, Berlin-New York, 1975. vi+224 pp.

[23] Lebowitz, J.L.; Rose, H.A.; Speer, E.R. Statistical mechanics of the nonlinear Schrödinger equation, J. Statist. Phys. 50 (1988), no. 3-4, 657-687.

[24] Ledoux, M.; Talagrand, M. Probability in Banach spaces. Isoperimetry and Processes, Ergebnisse der Mathematik und ihrer Grenzgebiete (3) [Results in Mathematics and Related Areas (3)], 23. SpringerVerlag, Berlin, 1991. xii+480 pp.

[25] Molinet, L. On ill-posedness for the one-dimensional periodic cubic Schrödinger equation, Math. Res. Lett. 16 (2009), no. 1, 111-120.

[26] Oh, T. Invariant Gibbs measures and a.s. global well posedness for coupled KdV systems, Differential Integral Equations 22 (2009), no. 7-8, 637-668.

[27] Oh, T. Invariance of the Gibbs measure for the Schrdinger-Benjamin-Ono system, SIAM J. Math. Anal. $41(2009 / 10)$, no. 6, 2207-2225.

[28] Oh, T. Invariance of the white noise for KdV, Comm. Math. Phys. 292 (2009), no. 1, 217-236. Also, see Erratum: "Invariance of the white noise for $K d V$ ", in preparation.

[29] Oh, T. White noise for KdV and $m K d V$ on the circle, RIMS Kôkyûroku Bessatsu B18 (2010), 99-124.

[30] Oh, T. Remarks on nonlinear smoothing under randomization for the periodic KdV and the cubic Szegö equation, to appear in Funkcial. Ekvac.

[31] Oh, T.; Quastel, J.; Valkó, B. Interpolation of Gibbs measures with white noise for Hamiltonian PDE, arXiv:1005.3957v1 [math.PR].

[32] Oh, T.; Sulem, C. On the one-dimensional cubic nonlinear Schrödinger equation below $L^{2}$, to appear in Kyoto J. Math.

[33] Paley, R.; Zygmund, A. On some series of functions (1), (2), (3), Proc. Camb. Phil. Soc. 26, (1930), 337-357; 26, (1930), 458-474; 28, (1933), 190-205.

[34] Quastel, J.; Valkó, B. KdV preserves white noise, Comm. Math. Phys. 277 (2008), no. 3, $707-714$.

[35] Thomann, L. Random data Cauchy problem for supercritical Schrödinger equations. Ann. Inst. H. Poincaré Anal. Non Linéaire 26 (2009), no. 6, 2385-2402. 
[36] Tsutsumi, Y. $L^{2}$-solutions for nonlinear Schrödinger equations and nonlinear groups, Funkcial. Ekvac. 30 (1987), no. 1, 115-125.

[37] Tzvetkov, N. Invariant measures for the nonlinear Schrödinger equation on the disc, Dyn. Partial Differ. Equ. 3 (2006), no. 2, 111-160.

[38] Tzvetkov, N. Invariant measures for the defocusing nonlinear Schrödinger equation, Ann. Inst. Fourier (Grenoble) 58 (2008), no. 7, 2543-2604.

[39] Tzvetkov, N. Construction of a Gibbs measure associated to the periodic Benjamin-Ono equation, Probab. Theory Related Fields 146 (2010), no. 3-4, 481-514.

[40] Zhidkov, P.E. An invariant measure for a nonlinear wave equation, Nonlinear Anal. 22 (1994), no. 3, 319-325.

[41] Zhidkov, P.E. Korteweg-de Vries and nonlinear Schrödinger equations: qualitative theory, Lecture Notes in Mathematics, 1756. Springer-Verlag, Berlin, 2001. vi+147 pp.

James Colliander, Department of Mathematics, University of Toronto, 40 St. George St, Toronto, ON M5S 2E4, CANADA

E-mail address: colliand@math.toronto.edu

Tadahiro Оh, Department of Mathematics, University of Toronto, 40 St. George St, TORONTO, ON M5S 2E4, CANADA

Current address: Department of Mathematics, Princeton University, Fine Hall, Washington Rd, Princeton, NJ 08544-1000, USA

E-mail address: hirooh@math.princeton.edu 